\title{
Top-k User-Defined Vertex Scoring Queries in Edge-Labeled Graph Databases
}

\author{
FRANCESCO PARISI, University of Calabria, Italy \\ NOSEONG PARK, University of North Carolina, USA \\ ANDREA PUGLIESE, University of Calabria, Italy \\ V. S. SUBRAHMANIAN, Dartmouth College, USA
}

\begin{abstract}
We consider identifying highly ranked vertices in large graph databases such as social networks or the Semantic Web where there are edge labels. There are many applications where users express scoring queries against such databases that involve two elements: (i) a set of patterns describing relationships that a vertex of interest to the user must satisfy and (ii) a scoring mechanism in which the user may use properties of the vertex to assign a score to that vertex. We define the concept of a partial pattern map query (partial PM-query), which intuitively allows us to prune partial matchings, and show that finding an optimal partial PM-query is NP-hard. We then propose two algorithms, PScore_LP and PScore_NWST, to find the answer to a scoring (top- $k$ ) query. In PScore_LP, the optimal partial PM-query is found using a list-oriented pruning method. PScore_NWST leverages node-weighted Steiner trees to quickly compute slightly sub-optimal solutions. We conduct detailed experiments comparing our algorithms with (i) an algorithm (PScore_Base) that computes all answers to the query, evaluates them according to the scoring method, and chooses the top- $k$, and (ii) two Semantic Web query processing systems (Jena and GraphDB). Our algorithms show better performance than PScore_Base and the Semantic Web query processing systems-moreover, PScore_NWST outperforms PScore_LP on large queries and on queries with a tree structure.
\end{abstract}

CCS Concepts: • Information systems $\rightarrow$ Data structures; Information retrieval query processing; Retrieval models and ranking;

Additional Key Words and Phrases: Graph databases, scoring queries, top- $k$ querying

\section{ACM Reference format:}

Francesco Parisi, Noseong Park, Andrea Pugliese, and V. S. Subrahmanian. 2018. Top-k User-Defined Vertex Scoring Queries in Edge-Labeled Graph Databases. ACM Trans. Web 12, 4, Article 21 (September 2018), 35 pages.

https://doi.org/10.1145/3213891

F. Parisi and N. Park equally contributed to the paper.

Some of the authors may have been partly funded by ONR Grants No. N000141612739, No. N000141512007, No. N000141612896, and No. N000141512742, by ARO Grant No. W911NF1410358, by the Start-(H)Open POR Grant No. J28C17000380006 funded by the Calabria Region Administration, and by the NextShop PON Grant No. F/050374/01-03/X32 funded by the Italian Ministry for Economic Development.

Authors' addresses: F. Parisi and A. Pugliese, DIMES Department, University of Calabria, Cubo 42C, Rende, Italy; emails: fparisi@dimes.unical.it, andrea.pugliese@unical.it; N. Park, Research Hall, Suite 417, George Mason University, 4400 University Drive, Fairfax, VA 22030-4422; email: npark2@uncc.ed; V. S. Subrahmanian, Dept. of Computer Science, Dartmouth College, 9 Maynard Street, Hanover, NH 03755; email: vs@dartmouth.edu.

Permission to make digital or hard copies of all or part of this work for personal or classroom use is granted without fee provided that copies are not made or distributed for profit or commercial advantage and that copies bear this notice and the full citation on the first page. Copyrights for components of this work owned by others than ACM must be honored. Abstracting with credit is permitted. To copy otherwise, or republish, to post on servers or to redistribute to lists, requires prior specific permission and/or a fee. Request permissions from permissions@acm.org.

(c) 2018 ACM 1559-1131/2018/09-ART21 $\$ 15.00$

https://doi.org/10.1145/3213891

ACM Transactions on the Web, Vol. 12, No. 4, Article 21. Publication date: September 2018. 


\section{INTRODUCTION}

Edge-labeled graph databases are becoming increasingly important. Many online social networks can be viewed as edge-labeled graphs. For instance, in LinkedIn, users are vertices. An edge $(u, v)$ from user $u$ to user $v$ may exist with a friend label if $u$ friended $v$ (and $v$ accepted), a pfriend label if $u$ friended $v$ but was not accepted, and a host of labels endorsed- $t$ if $u$ endorsed $v$ w.r.t. topic $t$ and not_endorsed- $t$ if $u$ was given the option of endorsing $v$ for topic $t$ but did not do so. Likewise, LinkedIn may also consider companies and employers to be vertices, universities, and schools to be vertices, and place edges between persons and such vertices labeled with relationships like employs or alumnus_of with the obvious meaning. On Twitter, vertices might include user handles, locations (from profiles), and hashtags that either appear on people's profiles or tweets. A follows edge from $u$ to $v$ might indicate that $u$ follows $v$, a location edge from $u$ to a location indicates that $u$ is from that location, and an interest edge from $u$ to $v$ might indicate that $u$ is interested in hashtag $v$. Thus, edge-labeled graphs can play an important role in capturing many of the semantic relationships explicitly mentioned in many social networks. Likewise, the (subject, property, object) triples used in RDF can be viewed as edge-labeled graphs where there is an edge from a subject to an object vertex.

While there has been extensive work on top- $k$ queries on ordinary graphs $[11,15,42,52]$, there has been no work on top- $k$ queries on edge-labeled graphs where the user gets to choose what is considered important when she is articulating her query, i.e., the concept of importance is expressed in the user query-in contrast, in most past work, the system imposes an a priori definition of importance. This capability of expressing importance-scoring methods within the query provides huge flexibility and capability to the user, and tends to be hard to index because we do not know, a priori, what the user's query and scoring method will be.

\subsection{Motivating Examples}

Consider a simple nuclear proliferation example in which an analyst is trying to identify suspicious entities. The analyst already has a network consisting of vertices (people, companies) and links between them. For instance, SPINN [1] extracts this network by first finding individuals/companies who have been sanctioned by the US and other similar governments for export control violations. SPINN then uses LinkedIn and Bloomberg's worldwide lists of directors and officers of companies to flesh this network out completely. Of course, not everyone in the SPINN's network of over 74K vertices and over $1 \mathrm{M}$ edges is necessarily bad. The suspiciousness level is represented as one of $\{1,0,-1\}$-each represents "suspicious," "unknown," and "non-suspicious," respectively.

Our knowledge of nuclear proliferation networks that have been previously discovered suggests some typical suspicious connections among suspect vertices. Two anonymized example pattern queries created from them are shown in Figure 1, one corresponding to a suspicious financial network, and another corresponding to a suspicious logistics network-both these can be illicit networks because money and materials must both be "moved" to achieve a desired goal. The query $P Q_{1}$ looking for suspicious financial networks starts by focusing on the suspicious bank B1-which has come to the notice of nuclear regulators for a reason. They are looking for banks matched for ? $b 1$ (through a path of "partner" links of maximum length 2), which has financed an energy company ? $c 2$ and a metallurgy company ? $c 1$. Such a subnetwork might be suspicious because bank $? b 1$ is financing nuclear supply producers. $P Q_{2}$, similarly, is looking for logistics companies $? c 1$ and $? c 2$ that are partners of the suspicious logistics companies $\mathrm{C} 7$ and $\mathrm{C} 8$, as well as two people ?p1, $? p 2$, and a bank ? $b 1$. Bank ? $b 1$ must have funded both ?c1 and ?c2. ?p1 and ?p2 must have friendship and are both linked to the suspicious logistics companies. If the same bank appears as a solution to $? b 1$ in both queries, then it would naturally be of greater interest to the analyst. 


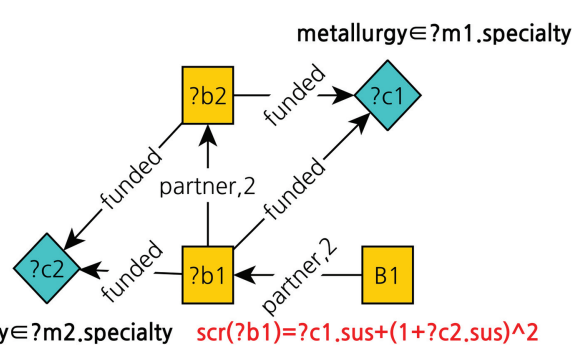

(a) $P Q_{1}$

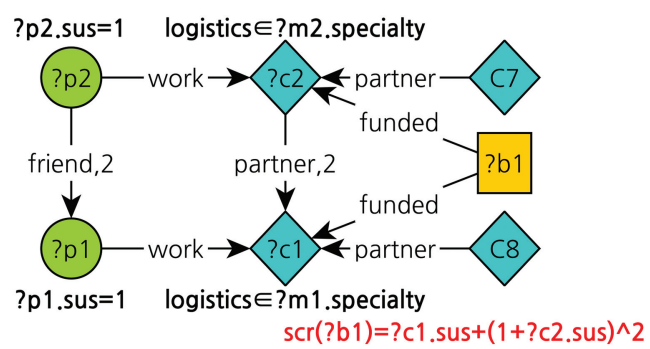

(b) $P Q_{2}$

Fig. 1. Example query. Circles represent people, diamonds represent companies, and rectangles represent banks. An edge annotated with an integer number $w$ can be matched to paths with maximum length $w$.

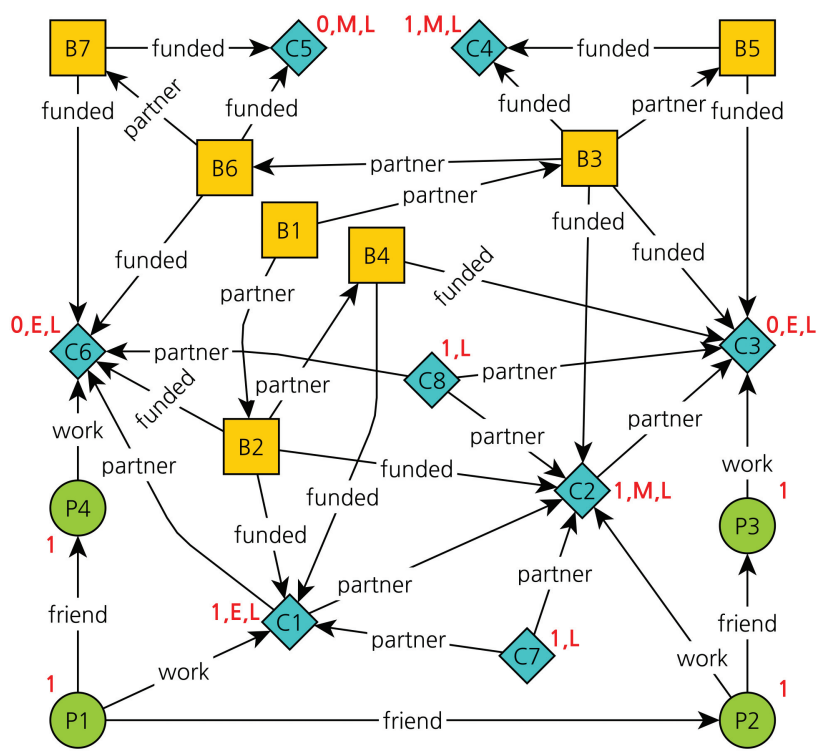

Fig. 2. Example graph database $\mathcal{G}$. Vertices are annotated with the values of their suspicious and specialty properties; integers represent suspiciousness levels; $M$ stands for metallurgy, L stands for logistics, and $E$ stands for energy.

To this end, we score matched patterns of each query with ?c1.sus $+(1+? c 2 . s u s)^{2}$ and project to ? $b 1$. In both queries, ? $c 2$ plays a more important role for nuclear proliferation. A vertex score in each query is then defined as an aggregated value (we use SUM in the example) over the scores of all patterns projected to the same vertex. Finally, we total the vertex score of each query up. With the simple SPINN network example of Figure 2, one pattern of $P Q_{1}$ maps $? b 1$ to $B 2-? b 2$ is mapped to $\mathrm{B} 4, ? c 1$ to $\mathrm{C} 2$ and $? c 2$ to $\mathrm{C} 1$, so $\mathrm{B} 2$ 's score is $1+(1+1)^{2}=5$ for $P Q_{1}$. Two patterns of $P Q_{2}$ map ? $b 1$ to $\mathrm{B} 2$. In the first one, ?c1 is mapped to $\mathrm{C} 6, ? c 2$ to $\mathrm{C} 1$, ?p1 to $\mathrm{P} 4$, and ?p2 to $\mathrm{P} 1$, so $\mathrm{B} 2$ 's score is $0+(1+1)^{2}=4$. In the second one, ?c1 is mapped to $\mathrm{C} 2$, ?c2 to $\mathrm{C} 1, ? p 1$ to $\mathrm{P} 3$, and ?p2 to $\mathrm{P} 1$, so B2's score is $1+(1+1)^{2}=5$. Thus, the overall score of B2 w.r.t. $P Q_{2}$ is 9 , and its score is 14 if we use SUM to aggregate scores (the scores of B3, B4, and B6 are 6, 4, and 1, respectively).

Queries such as this require the following features: (i) The user must be able to specify a set of patterns of interest to her. (ii) The user must be able to specify a scoring function that extracts properties of a vertex (?c1.sus above) and use it to define a scoring metric. (iii) The user must be 

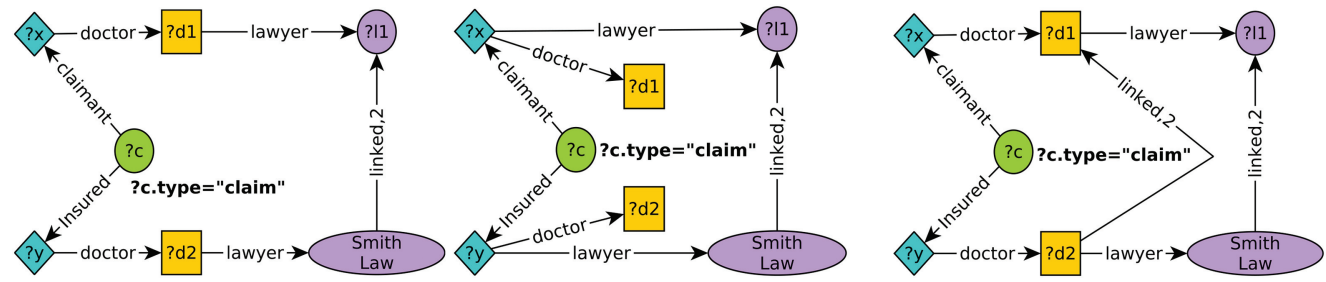

Fig. 3. Example query patterns for the medical insurance fraud scenario.

able to specify maximum path lengths in her query. (iv) The user must be able to "bump up" the score of a vertex that satisfies multiple criteria by aggregating them (as is done by adding up the two scores above).

The use of patterns similar to those in the SPINN nuclear trafficking application applies in many different commercial fraud investigations as well. For instance, one type of medical insurance fraud (MIF) involves staging fake accidents. The individuals and organizations involved in such MIF networks involve a network of individuals (called "runners") who stage accidents and bring fake "victims" to one or more "clinics." Another network of individuals handle the legal side of things-they involve networks of lawyers and claimants who work closely together in the scam. A third network of individuals include the medical professionals and doctors who deliver the medical services-doctors involved in the fraud are often connected with other doctors. And a fourth network ties the clinics involved that ultimately bill the insurance company. Often times, the same patient visits many different clinics, and the same doctors are tied to different clinics involved in the fraud, and so are the lawyers. A person who ends up occurring in many of these networks is generally more suspicious than others. Insurance investigators wish to specify methods to focus on the most suspicious individuals-something that can be expressed via the scoring queries (Squeries) proposed in this article-and they are generally looking for the top- $k$ suspicious people as they are limited in their investigative resources. ${ }^{1}$ Because the MIF pattern is so complex, there are a whole range of possible graph patterns that fraud investigators might look for. Figure 3 shows three such graph patterns. The first one wishes to find lawyers (?l1) and doctors (?d1,?d2) who are involved in claims and are linked to the Smith law firm, which may be suspicious for various reasons. The second and third patterns are different patterns that are also looking for doctors and lawyers linked in a different way to a different law firm.

If the same lawyer and/or the same doctors show up many different times in the answers to one or more of these patterns, they have to be considered more suspicious. For instance, the same doctor or the same lawyer might be involved in many different claims. In such cases, we would like to assign a higher score to the ?l1, ?d1, ?d2 variables. One way an investigator might do this is by defining the score of such variables as $\frac{? c . c l a i m \_a m o u n t}{10 \mathrm{~K}}+? x$.work $\_$years, where $? x \in\{? l 1, ? d 1, ? d 2\}$-this scoring function also considers a person's skills in her area of work.

And of course, if the same doctor/lawyer shows up as an answer across more than one of these three patterns, she has to be considered even more suspicious. Hence, the investigator could define the final score of the doctor or lawyer as the sum of their scores across the different patterns.

In general, the fraud investigator has limited resources to carry out physical and/or electronic surveillance of these suspicious entities. She wishes to assign a suspicion score to doctors/lawyers so she can focus her limited resources on those most likely involved in the fraud. This is why

\footnotetext{
${ }^{1}$ See http://linkanalysisnow.com/2009/04/analyzing-medical-fraud.html for a detailed analysis of how such scams work. This site also provides links to lots of other fraudulent activities where scoring queries may play a natural role.
} 
she uses the scoring functions shown above to capture her suspicion of the different doctors and lawyers known to the insurance company.

The above two examples, one each on identifying suspicious vertices in nuclear proliferation investigations, as well as in identifying suspicious vertices in MIF, are special cases of broader investigations into different types of illicit activity. The same principles apply to other types of complex crime networks such as money laundering networks, drug networks, human trafficking networks, to name a few. Investigations to identify suspicious vertices in all of these networks are critical because there are limited resources for carrying out physical and/electronic surveillance. In some cases, they are downright dangerous (e.g., to place an undercover officer in the illicit network). This is why the complexity of the query language is not important from an application perspective-rather, the query language must allow the investigator to articulate precisely the patterns of interest to her, together with her own methods for scoring. Simply put, lives may depend on it, at least in such applications.

As it will be discussed in detail in Section 2, many of the query models proposed in the past do not perform aggregation but rather concentrate on individual patterns-our model is conceptually more similar to SQL queries with both GROUP BY and ORDER BY clauses. The different existing models obviously have different possible applications-however, costly algorithms are usually needed to process GROUP BY queries. One key aspect of our proposed model is also its capability to handle graph queries equipped with very flexible scoring functions, so that users are able to conduct more sophisticated pattern-matching tasks such as the ones described above.

\subsection{Plan of the Article}

The rest of the article is organized as follows. Section 2 describes related work. Then, in Sections 3 and 4 , we define graph databases, propose $S$-queries that capture the above features, and define the top- $k$ answers to S-queries. Section 5 describes our S-query processing algorithm. The algorithm includes a method to estimate an upper bound on the score of any vertex that allows us to prune the search for top- $k$ answers. The estimation of these upper bounds is critical, but unfortunately, exactly finding these upper bounds is NP-hard. Because of this, we present two algorithms: the PScore_LP algorithm where the optimal upper bound is found using a list-oriented pruning method, and the PScore_NWST algorithm, which leverages node-weighted Steiner trees (NWSTs) to quickly find very good upper bounds (though they may not be optimal). Section 6 discusses how our approaches can be extended to encompass any maximum path length constraints expressed in the query. Section 7 summarizes our experiments on seven real-world datasets involving graphs with up to $234 \mathrm{M}$ edges.

\section{RELATED WORK}

Subgraph matching has been extensively studied [11, 18, 31, 35, 42, 49, 54]-surveys of the work in this field can be found, e.g., in References [27], [29], [22].

Some approaches (e.g., References [11,42]) map edges in the query to paths in the graph-these approaches may constrain path lengths but do not try to identify top- $k$ vertices. Instead, they focus on finding patterns in the graph data that are isomorphic to a given query pattern-the isomorphisms yield substitutions that assign vertices in the graph to variables in the expressed patterns. We leverage some of these works in this article in terms of the edge to path mappings, as we allow single query edges to be mapped to paths in the graph. Others try to find the top- $k$ substitutions in different situations. The HRJoins proposed in Reference [42] are targeted at twig queries on graph data, in which substitutions are scored (not vertices as we do). The score of a substitution is the sum of the edge weights of all edges involved in the edge to path mappings. The concept of kGPM queries is used in Reference [11] to extend this work to graph data. They score answer 
substitutions using the sum of edge weights. The $k$ most similar subgraphs to a query graph, where similarity is defined using maximum common subgraphs, are targeted in Reference [54] with the introduction of the DPIndex, OPIndex, and GSIndex structures.

In the LONA framework proposed in Reference [52] for top- $k$ queries, the user does not specify patterns, but vertices in the graph are scored via a function $f$. This is a relevance score for each vertex. Once these relevance scores are computed, the authors compute a higher-level score that aggregates the lower-level relevance scores of a vertex and all its neighbors within some number of hops specified in the query. The aggregation is computed as $\sum_{u \in N b r(v, h)} f(u)$, where $\operatorname{Nbr}(v, h)$ is a set of $h$-hop neighbors of $v$. Top- $k$ vertex set search by graph simulation has been suggested in Reference [15], where the score of the output variable of a single-pattern query is defined as the size of the maximum graph simulation matching the output variable to a vertex. Both the above approaches do not allow the user to specify multiple patterns in the query nor to choose the scoring function.

The concept of graph database used in this article is similar to that of the Heterogeneous Information Network (HIN) [48], which is modeled as a directed graph where vertices and edges are annotated with types. In a graph database, each vertex is associated with multiple properties, including type, and edges are associated with labels that can be viewed as types. Thus, graph databases generalize HINs by allowing multiple properties to be associated with vertices. A relevant problem investigated in HINs is measuring similarity between vertices [44]. In this regard, using the "meta-paths" semantics (i.e., paths defined on the schema-level graph of the vertices' types), Reference [48] proposed PathSim, a meta-path-based similarity measure for evaluating the similarity of same-typed vertices based on symmetric paths. Meta-paths are a special case of metastructures [24] that consist of a direct acyclic (schema-level) graph with a single source vertex and a single target vertex. In Reference [24], to measure the relevance of two vertices, measures are proposed that are based on looking at the number of sub-graphs that match a meta-structurethis is done through sub-graph expansion restricted to a meta-structure. Symmetric metagraphs similar to meta-structures are exploited in Reference [16], where a supervised approach to proximity search is proposed. They aim at learning weights for optimally aggregating different characteristic metagraphs that specify classes of semantically similar objects. This can be viewed as aggregating multiple query patterns, but the end-user does not take part in the process, which is designed to automatically learn weights for combining different metagraphs. Different from the above-mentioned approaches, we do not rank vertexes by measuring their proximity to a query vertex in terms of the (weighted) numbers of meta-paths instances, but compute top- $k$ answers on the basis of user-defined (local and global) aggregation functions that are evaluated on vertices once the patterns provided in the query are fully mapped to the graph data.

The MAGE system proposed in Reference [41] supports exact and approximate subgraph matching on graphs with both vertex and edge attributes, for which wildcards and multiple values attributes are allowed. The $k$ desired matching subgraphs are ranked through a (fixed) proximity measure based on multiplying the RWR (Random Walk with Restart) values of the pairs of vertices in the graph that instantiate pairs of vertices in the query. Finally, the graph-querying framework NeMa proposed in Reference [28] allows for ambiguity in both the structure and vertex labels. Instead of checking graph isomorphism, NeMa identifies top- $k$ optimal matches by minimizing vertex labels differences (using Jaccard similarity measure) and vertex pairs distances. Thus, it is designed to possibly return results that deviate from the given query pattern.

It is also worth noting the importance of handling multiple query patterns "by design." In fact, the user brings her own preferences and knowledge during the whole process of query definitionfrom the definition of the local scores for the query patterns she believes are more significant, to the choice of a global function for aggregating single patterns' scores. This is a simple, yet crucial 
difference with some of the approaches discussed above. Indeed, though, in principle, existing techniques could handle multiple query patterns by processing single query patterns one by one and then aggregating the scores in a custom way, there are several issues that can make it relatively impractical for the user to understand the meaning the final answer obtained. In fact, most of the other approaches define the (local) scores of top- $k$ answers of single query patterns by ranking the obtained substitutions through predefined functions that the user is supposed to use as a black box (for instance, RWR multiplication in MAGE). Moreover, it is in general not easy to determine the $k^{\prime}$ value the user should use to extract top- $k^{\prime}$ intermediate answers to single patterns and then build the final top- $k$ answers over multiple patterns. To obtain this, existing techniques may need to apply "exhaustive" approaches based on the maximum possible value of $k^{\prime}$ for the intermediate answers. To see why, consider for instance a simple case where the (local) scores obtained using two single query patterns are $s_{1}=\{5,3,2\}$ and $s_{2}=\{3,2,1\}$, respectively, and we are interested in extracting the top-1 answer obtained by averaging local scores. In this case, taking the average of any top- $k^{\prime}$ subset of $s_{1}$ or $s_{2}$, for any $k^{\prime}<3$, would result in a wrong top-1 final score.

As a final remark, we point out that relevant work has focused on the storage and querying of RDF data $[2-5,8,10,14,19,20,26,38,40,43,51,56]$ and its associated SPARQL query language [23]. Our proposed vertex importance queries can be expressed in SPARQL through the use of GROUP BY, UNION, LIMIT, and ORDER BY operators, and by "expanding" queries into equivalent sets of queries without edge length constraints (SPARQL "property paths" do not allow such constraints). However, the majority of the approaches to RDF storage and querying are based on traditional relational storage techniques that are known to have lower performance than graph databases when answering subgraph matching queries, due to the large number of joins needed on the tables representing the structure of the graph $[2,10,43]$. In particular, the approaches used in many existing RDF query engines (e.g., References [8, 26, 47, 51]) severely limit the actual usability of property paths, due to the huge computational effort needed to join the tables built [2]. Neo4j [36], Titan [50], and DOGMA [7] (Titan's original research work) provide (recursive) graph-traversal subgraph-matching algorithms based on proprietary graph indexing schemes (to avoid table joins). We employ the DOGMA algorithm as a basis for our mapping algorithm. Specific top- $k$ query processing algorithms for RDF or social network data have been proposed as well (e.g., References [32], [39], [53], and [55]). However, they are mainly interested in scoring substitutions (or projections of substitutions) without aggregation. In particular, the ranked matching (RM) algorithm [55] scores substitutions of a query pattern by summing up, for each vertex $v$ of a query, the pairwise similarity between $v$ and its matching in the graph. A function providing the similarity values, for each pair of vertices in the graph, is assumed as input of the algorithm, and simple data graphs without labels are considered. Attributed graphs are instead considered in Reference [39], where OptIQ (Optimized Importance Query) algorithm is introduced for computing top- $k$ answers that are obtained by ranking substitutions with user-defined scoring functions without aggregation. The STAR framework [53] reduces the computation of top- $k$ answers to a (general) graph query to the computation of the answers to a set of star-shaped queries. The top- $k$ answers to each star query are defined by ranking substitutions, each of them scored by summing up the similarity scores of the vertices and edges that match the query pattern. Specifically, weighted aggregation of different similarity functions (provided by the system, not by the end-users) can be used to define the score of each vertex, and matching a single edge to a path of bounded length is allowed. In general, the above approaches score substitutions (or projections of substitutions) without aggregation-thus, they only focus on ORDER BY and LIMIT queries that suffice for their purpose.

The features of some of the above related works are summarized in Table 1, where, in the "top- $k$ " column, symbol V (resp. S) means that that work addresses the problem of identifying the top- $k$ vertices (resp. substitutions). 
Table 1. Comparison with Related Work

\begin{tabular}{|c|c|c|c|c|c|}
\hline & $\begin{array}{c}\text { Multiple } \\
\text { patterns }\end{array}$ & $\begin{array}{c}\text { Vert. prop.- } \\
\text { based scoring }\end{array}$ & $\begin{array}{c}\text { Path } \\
\text { edges }\end{array}$ & $\begin{array}{c}\text { Global } \\
\text { scoring }\end{array}$ & Top- $k$ \\
\hline \hline kGPM [11] & - & - & $\sqrt{ }$ & - & $\sqrt{ }(\mathrm{S})$ \\
HRJoins [42] & - & - & $\sqrt{ }$ & - & $\sqrt{ }(\mathrm{S})$ \\
Simulation [15] & - & - & $\sqrt{ }$ & - & $\sqrt{ }(\mathrm{V})$ \\
LONA [52] & - & $\sqrt{ }$ & - & - & $\sqrt{ }(\mathrm{V})$ \\
DPInd., OPInd., & & & & & \\
GSInd. [54] & - & - & - & - & $\sqrt{ }(\mathrm{S})$ \\
PathSim [48] & - & - & - & - & $\sqrt{ }(\mathrm{S})$ \\
Meta Structure [24] & - & - & - & - & $\sqrt{ }(\mathrm{S})$ \\
Meta Graph [16] & $\sqrt{ }$ & - & - & $\sqrt{ }$ & $\sqrt{ }(\mathrm{S})$ \\
MAGE [41] & - & - & - & - & $\sqrt{ }(\mathrm{S})$ \\
NeMa [28] & - & - & - & - & $\sqrt{ }(\mathrm{S})$ \\
STAR [53] & - & - & $\sqrt{ }$ & - & $\sqrt{ }(\mathrm{S})$ \\
RM [55] & - & - & - & - & $\sqrt{ }(\mathrm{S})$ \\
OptIQ [39] & - & $\sqrt{ }$ & - & - & $\sqrt{ }(\mathrm{S})$ \\
This work & $\sqrt{ }$ & $\sqrt{ }$ & $\sqrt{ }$ & $\sqrt{ }$ & $\sqrt{ }(\mathrm{V})$ \\
\hline
\end{tabular}

\section{GRAPH DATABASES}

We assume the existence of four arbitrary, but fixed, disjoint sets $V, \mathcal{L}, \mathcal{P}, \mathcal{T}$ of vertex names, edge labels, vertex properties, and type names, respectively. Each $p \in \mathcal{P}$ has an associated domain $\operatorname{dom}(p)$, which is a set (disjoint from $V, \mathcal{L}$, and $\mathcal{P}$ ) of values that can be assigned to $p$. We assume the existence of a special property type $\in \mathcal{P}$ with domain $\mathcal{T}$. A graph database is defined as follows.

Definition 3.1 (Graph Database). A Graph Database $\mathcal{G}$ is a triple $\langle V, E, \wp\rangle$, where

$-E \subseteq V \times \mathcal{L} \times V$ is a set of labeled edges;

$-\wp: V \times \mathcal{P} \rightarrow \bigcup_{p \in \mathcal{P}} \operatorname{dom}(p)$ is a (possibly partial) property assignment function, such that (i) if $\wp(v, p)$ is defined, then $\wp(v, p) \in \operatorname{dom}(p)$, and (ii) $\wp(v, t y p e)$ is defined for all $v \in V .^{2}$

In our running example, the set of types may include person, company, bank, and more. A sample graph database is shown in Figure 2. If $\mathcal{P}=\{$ type, suspicious, specialty $\}$ and dom(type $)=$ $\{$ person, company, bank\}, the property function assigns the value person to the pair (P1, type), meaning that person is the type of the vertex named $P 1$.

In the remainder of the article, we assume an arbitrary but fixed graph database $\mathcal{G}=\langle V, E, \wp\rangle$ is given.

\section{SCORING QUERIES}

In this section, we formally define S-queries, an example of which was informally given in the Introduction. An S-query consists of a set of Pattern Map Queries (PM-queries) as shown by the graphs in Figure 1 and a global aggregation function, according to which the scores of answers generated by the individual PM-queries can be evaluated. Intuitively, a PM-query is used to specify a way to (locally) score a class of sub-graphs of $\mathcal{G}$ having some characteristic (structure, vertex properties,

\footnotetext{
${ }^{2}$ We assume that every vertex has a type property because vertices have types (or types can be inferred) in the majority of real world scenarios, and this is a valuable additional information used by our index structure (see Section 5.2). In the absence of types, we can simply assign a unique fictitious type to all vertices in the database and queries.
} 
Table 2. Symbols Used

\begin{tabular}{|c|c|c|}
\hline Symbol & Section & Meaning \\
\hline$\wp$ & 3 (Definition 3.1) & Property assignment function \\
\hline $\mathcal{G}=\langle V, E, \wp\rangle$ & 3 (Definition 3.1) & Graph database \\
\hline$\chi$ & 4.1 (Definition 4.1) & Set of constraints over variables \\
\hline$\varrho$ & 4.1 (Definition 4.1) & $\begin{array}{l}\text { Function that associates variables with } \\
\text { numerical terms }\end{array}$ \\
\hline la & 4.1 (Definition 4.1) & Local aggregation function \\
\hline$P Q=\left\langle V_{P Q}, E_{P Q}, \chi, \varrho, l a\right\rangle$ & 4.1 (Definition 4.1) & PM-query \\
\hline$g a$ & 4.1 (Definition 4.2) & Global aggregation function \\
\hline$S Q=\langle P Q S, g a\rangle$ & 4.1 (Definition 4.2) & $\mathrm{S}$-query ( $P Q S$ is a set of $\mathrm{PM}$-queries) \\
\hline$l s c o r e(v, P Q)$ & 4.1 (Definition 4.4) & Local score of $v$ w.r.t. $P Q$ \\
\hline $\operatorname{score}(v, S Q)$ & 4.1 (Definition 4.5) & Score of $v$ w.r.t. $S Q$ \\
\hline $\operatorname{ans}(S Q)$ & 4.1 (Definition 4.6) & Top- $k$ answer to $S Q$ \\
\hline $\mathcal{E}(P Q)$ & 5.1 & Set of essential variables in $P Q$ \\
\hline$P P Q$ & 5.1 (Definition 5.1) & Partial PM-query for $P Q$ \\
\hline $\operatorname{score}^{*}(v)$ & 5.2 & Upper bound on $\operatorname{score}(v, S Q)$ \\
\hline$s u b^{*}(P Q)$ & 5.2 & $\begin{array}{l}\text { Upper bound on the number of answer } \\
\text { substitutions for } P Q\end{array}$ \\
\hline $\begin{array}{r}\operatorname{cand}(v, \delta, \pi,(l, d)) \\
\operatorname{cand}^{*}(P Q, ? x)\end{array}$ & 5.2 & $\begin{array}{l}\text { Number of vertices that are connected to } v \\
\text { via an undirected path of length } \delta \text { ending with } \\
\text { an edge labeled } \ell \text { of type } \pi \text { with direction } d \\
\text { Upper bound on the number of substitutions } \\
\text { for variable } ? x \text { in } P Q\end{array}$ \\
\hline $\operatorname{opt}(P Q)$ & 5.3 & Chosen partial PM-query for $P Q$ \\
\hline$s u b^{*}(P P Q, P Q)$ & 5.3 & $\begin{array}{l}\text { Upper bound on the number of substitutions } \\
\text { for the variables in } P Q \text { that are not in } P P Q\end{array}$ \\
\hline PScore & 5.4 & General query processing algorithm \\
\hline PScore_LP & 5.4 & PScore algorithm with list-oriented pruning \\
\hline PScore_NWST & 5.4 & PScore algorithm with NWST-based pruning \\
\hline$e q(P Q)$ & 6.1 & $\begin{array}{l}\text { Equivalent set of non-annotated "expanded" } \\
\text { PM-queries for } P Q\end{array}$ \\
\hline $\operatorname{cov}(P P Q)$ & 6.1 & $\begin{array}{l}\text { Subset of eq(PQ) such that, by extending } \\
\text { a substitution for } P P Q \text {, full substitutions for } \\
\text { all the queries in the subset can be obtained }\end{array}$ \\
\hline
\end{tabular}

or edge labels) the user wants to look for. Table 2 summarizes the symbols used throughout the article.

\subsection{Pattern Map Queries}

Let $V A R$ be a set of variable symbols (we use strings starting with question marks as for variables). We assume $V A R$ is disjoint from $V, \mathcal{L}, \mathcal{P}, \mathcal{T}$ and from $\bigcup_{p \in \mathcal{P}} \operatorname{dom}(p)$. The idea is that variables must be "bound" to vertices in the graph database. A (numerical) term is a value of a property, a property of a query variable, a real number, or a polynomial-time computable function of terms. For instance, $(? x . s u s-1),(? x . s u s+1) *(?$ y.sus +2$)$ are all terms. A constraint is a comparison between terms, the application of a set operator to terms, or the application of a logical operator $(\wedge, \vee$, or $\neg)$ to 
constraints. For instance, Energy $\in$ ?x.specialty, ?x.sus $>$ ?y.sus, and $(? x$. sus $>0 \wedge$ ?x.sus $\leq$ y.sus +1$)$ are all constraints.

We are now ready to introduce the notion of pattern map query (PM-query). Let $*$ be a distinguished "wildcard" label not occurring in $\mathcal{L}$.

Definition 4.1 (Pattern Map (PM) Query). A PM-query is a 5-tuple $P Q=\left\langle V_{P Q}, E_{P Q}, \chi, \varrho, l a\right\rangle$, where:

$-V_{P Q} \subseteq V \cup V A R$ with $V_{P Q} \cap V \neq \emptyset$.

$-E_{P Q} \subseteq V_{P Q} \times\left(2^{\mathcal{L}} \cup\{*\}\right) \times \mathbb{Z}^{+} \times V_{P Q}$.

$-\chi$ is a finite set of constraints over variables in $V_{P Q}$.

$-\varrho$ is a partial function that associates variables in $V_{P Q}$ with numerical terms.

$-l a \in\{M I N, M A X, A V G, S U M\}$ is a "local" aggregation function.

We assume that every variable in $V_{P Q}$ has a type constraint. In addition, if $\varrho(? x)$ is defined and its value is $t$, then we say that ? $x$ is an output variable, $t$ is a scoring term, and any variable occurring in $t$ is a scoring variable. Moreover, if $E_{P Q}$ contains an edge $e=\left(v, L, w, v^{\prime}\right)$ with $w>1$, then we say that $e$ is annotated and $P Q$ is edge-annotated.

The first two items of Definition 4.1 say that a PM-query is a directed edge-labeled graph where (i) each vertex is either a vertex name or a variable symbol and (ii) each edge is labeled with a pair consisting of a set of edge labels (or *) and an integer. Intuitively, the user uses this graph to specify the structure of the sub-graphs of $\mathcal{G}$ she is looking for (where $*$ matches any label), including constraints about the maximum length of paths. The constraints in $\chi$ express requirements about vertex properties. Function $\varrho$ provides a flexible way for ranking the vertices the user is interested in by means of (possibly complex) scoring terms, while $l a$ is a local aggregation function that tells us how scoring terms are aggregated. Finally, output variables are those that will be mapped to the vertices the user wants to compute local scores for, whereas scoring variables are those that will be mapped to the vertices that contribute to such scores.

It should be observed that we only consider anchored queries, that involve at least one constant. Anchored queries are considered of fundamental importance in many scenarios-for instance, anchors are present in (i) all queries in both the DBPedia [34] and LUBM [30] SPARQL benchmarks, (ii) 14 out of 20 queries in the interactive use case of the Social Network Intelligence BenchMark [45], (iii) 19 out of 20 queries in the explore and business intelligence use cases of the Berlin benchmark [6], and (iv) 14 out of 15 queries in the $S P^{2}$ Bench benchmark [46].

An S-query consists of a set of PM-queries along with a global aggregation function that combines the scores returned by each PM-query.

Definition 4.2 (Scoring Query). An S-query is a pair $S Q=\langle P Q S, g a\rangle$, where $P Q S$ is a finite set of $\mathrm{PM}$-queries and $g a \in\{M I N, M A X, A V G, S U M\}$ is a "global" aggregation function.

For instance, if we consider the PM-queries $P Q_{1}$ and $P Q_{2}$ of our running example, $S Q=$ $\left\langle\left\{P Q_{1}, P Q_{2}\right\}, S U M\right\rangle$ is an $\mathrm{S}$-query that ranks banks by the sum of the scores obtained from $P Q_{1}$ and $P Q_{2}$.

To define the answer to a PM-query and an S-query, we need some intermediate concepts.

A substitution $\theta$ is a mapping $\theta: V A R \rightarrow V$. Applying $\theta$ to an expression $e$ (vertex, term, or constraint) means replacing every variable $? x$ in $e$ with vertex name $\theta(? x)$, and the ground (i.e., variable-free) expression resulting from such an application is denoted $e \theta$. Ground terms and ground constraints are evaluated in the obvious way.

Definition 4.3 (Answer Subst. for a PM-query). Given a PM-query $P Q=\left\langle V_{P Q}, E_{P Q}, \chi, \varrho, l a\right\rangle$, an answer substitution $\theta$ for $P Q$ is a mapping $\theta: V_{P Q} \rightarrow V$ such that 
- for each $(\alpha, L, m, \beta) \in E_{P Q}$, there is path $\left(v_{1}, \ell_{1}, v_{2}\right),\left(v_{2}, \ell_{2}, v_{3}\right), \ldots,\left(v_{m^{\prime}}, \ell_{m^{\prime}}, v_{m^{\prime}+1}\right)$ in $\mathcal{G}$ with $m^{\prime} \leq m$ such that $v_{1}=\alpha \theta, v_{m^{\prime}+1}=\beta \theta$, and, for each $i \in\left[1 . . m^{\prime}\right], \ell_{i} \in L$ if $L \neq\{*\}$;

- for each constraint $C \in \chi, C \theta$ evaluates to true.

For instance, two answer substitutions for $P Q_{1}$ of our running example are $\{? b 1 / \mathrm{B} 3, ? c 1 / \mathrm{C} 4$, $? c 2 / \mathrm{C} 3, ? b 2 / \mathrm{B} 5\}$ and $\{? b 1 / \mathrm{B} 6, ? c 1 / \mathrm{C} 5, ? c 2 / \mathrm{C} 6, ? b 2 / \mathrm{B} 7\} .^{3}$

The existence of an answer substitution $\theta$ for a $P M$-query $P Q$ guarantees that there is a way of mapping all the variables in $P Q$ to vertices of $G$ so that it satisfies all other requirements specified by $P Q$ : presence of vertex names specified in the query, paths having a given maximum length with appropriate edge labels, and vertex properties satisfying the constraints.

Given an answer substitution $\theta$ for $P Q$, and an output variable ? $x$ of $P Q$, the score of a vertex $v=? x \theta$ in $\mathcal{G}$ can be computed by evaluating $\varrho(? x) \theta$, which results in a numerical value (obtained when the numeric term $\varrho(? x)$ is instantiated by $\theta$ ). Thus, if a vertex $v \in \mathcal{G}$ is the result of applying an answer substitution $\theta$ to a variable $? x \in V_{P Q}$, then it has a (local) score obtained by using $l a$ to aggregate all the values $\varrho(? x) \theta$. This is captured formally below.

Definition 4.4 (Local Score). Let $v$ be a vertex in $\mathcal{G}$, and $P Q=\left\langle V_{P Q}, E_{P Q}, \chi, \varrho, l a\right\rangle$ a PM-query. Let $S$ be the set $\{\varrho(? x) \theta \mid \theta$ is an answer substitution for $P Q$ and $v=? x \theta\}$. The local score of $v$ w.r.t. $P Q$, denoted $l \operatorname{score}(v, P Q)$, is $l a(S)$ if $S \neq \emptyset$, and undefined otherwise.

For instance, in our running example, lscore $\left(\mathrm{B} 2, P Q_{1}\right)=5$ and $l$ score $\left(\mathrm{B} 2, P Q_{2}\right)=9$.

Given an S-query $S Q=\langle P Q S, g a\rangle$ and a vertex $v$ of $\mathcal{G}$, we compute a single score for $v$ w.r.t. $S Q$ by aggregating (via the global aggregate function) the local scores of $v$ w.r.t. each PM-query $P Q \in P Q S$.

Definition 4.5 (Score). Let $v$ a vertex in $G$ and $S Q=\langle P Q S, g a\rangle$ an S-query. Let $S$ be the set $\{l \operatorname{score}(v, P Q) \mid P Q \in P Q S\}$. The score of $v$ w.r.t. $S Q$ is defined as $\operatorname{score}(v, S Q)=g a(S)$ if $S \neq \emptyset$, and undefined otherwise.

In our running example, $\operatorname{score}(\mathrm{B} 2, S Q)=14, \operatorname{score}(\mathrm{B} 3, S Q)=6$, and $\operatorname{score}(\mathrm{B} 4, S Q)=4$.

The top- $k$ answer to an S-query $S Q$ is given by the set of vertices of $\mathcal{G}$ having the $k$ highest scores.

Definition 4.6 (Top-k Answer to an S-query). Let $S Q=\langle P Q S, g a\rangle$ be an S-query and $k \leq|V|$ a positive integer. The top- $k$ answer to $S Q$ is the set ans $(S Q)=\left\{v_{1}, \ldots, v_{k}\right\} \subseteq V$ such that (i) for each $i \in[1 . . k-1]$, $\operatorname{score}\left(v_{i}, S Q\right) \geq \operatorname{score}\left(v_{i+1}, S Q\right)$; (ii) there is no vertex $v \in V \backslash \operatorname{ans}(S Q)$ such that $\operatorname{score}(v, S Q)>\operatorname{score}\left(v_{k}, S Q\right)$.

In our running example, the top-2 answer to $S Q$ is the set $\{\mathrm{B} 2, \mathrm{~B} 3\}$.

\section{QUERY PROCESSING}

To efficiently compute the top- $k$ answer to an S-query $S Q=\langle P Q S, g a\rangle$, we perform the following steps:

(1) We partially match the PM-queries in $P Q S$.

(2) We estimate upper bounds on the scores obtainable by vertices in $\mathcal{G}$.

(3) We then progressively extend these partial matches. In the process, vertices that are no longer candidates to be in the top- $k$ answer (since their upper bounds are lower than the current top- $k$ ) are pruned.

\footnotetext{
${ }^{3}$ We use multisets of substitutions in general, since two same substitutions with different intermediate edges between vertices, when edges are annotated, are counted twice [23].
} 
Table 3. Essential Partial Substitutions for $P Q_{1}$ and $P Q_{2}$ (without Annotations). $\theta_{i, j}$ Is the $j$-th Partial Substitution for $P Q_{i}$

\begin{tabular}{|c|c|c|c|}
\hline Substitution & $? b 1$ & $? c 1(? c 1 . s u s)$ & $? c 2(? c 2 . s u s)$ \\
\hline \hline$\theta_{1,1}$ & B3 & C4 (1) & C3 (0) \\
\hline$\theta_{1,2}$ & B3 & C2 (1) & C3 (0) \\
\hline$\theta_{1,3}$ & B2 & C2 (1) & C1 (1) \\
\hline$\theta_{1,4}$ & B2 & C2 (1) & C6 (0) \\
\hline$\theta_{2,1}$ & B2 & C6 (0) & C1 (1) \\
\hline$\theta_{2,2}$ & B2 & C2 (1) & C1 (1) \\
\hline$\theta_{2,3}$ & B4 & C2 (1) & C1 (1) \\
\hline$\theta_{2,4}$ & B3 & C3 $(0)$ & C2 (1) \\
\hline$\theta_{2,5}$ & B2 & C3 (0) & C2 (1) \\
\hline
\end{tabular}

In this section, we describe the techniques we developed to support the above process in the case of non-annotated queries. Later, in Section 6, we discuss the extensions for the case of edgeannotated queries.

\subsection{Partial Matching}

In our approach, partial matching is performed by computing substitutions for a subset of the variables in a given PM-query $P Q$. A query variable is said to be essential if it is either an output variable or a scoring variable in $P Q$. We use $\mathcal{E}(P Q)$ to denote the set of all essential variables in $P Q$. For instance, in both the PM-queries of our running example, ? $b 1, ? c 1$, and ?c2 are essential variables. Formally, a partial matching is the application of a substitution to a partial PM-query extracted from $P Q$ that includes a subset of vertices of $P Q$ (containing all essential variables) and a subset of edges of $P Q$, as defined below.

Definition 5.1 (Partial PM-query). Given a PM-query $P Q=\left\langle V_{P Q}, E_{P Q}, \chi, \varrho, l a\right\rangle$, a partial $P M$ query $P P Q$ for $P Q$ is a 5-tuple $P P Q=\left\langle V_{P P Q}, E_{P P Q}, \chi, \varrho, l a\right\rangle$ such that:

$-\mathcal{E}(P Q) \subseteq V_{P P Q} \subseteq V_{P Q}$

$-V_{P P Q} \cap V \neq \emptyset$;

$-E_{P P Q} \subseteq E_{P Q}$ is such that there exists at least one undirected path between every pair of essential variables in $\mathcal{E}(P Q)$, and at least one undirected path between each essential variable in $\mathcal{E}(P Q)$ and a constant vertex in $V_{P P Q}{ }^{4}$

As an example, $? c 1 \leftarrow ? b 1 \& ? c 2 \leftarrow ? b 1 \leftarrow \mathrm{B} 1$ is a partial PM-query for $P Q_{1}$ of our running example (we sometimes abuse notation and use this shorthand notation for brevity).

Definition 5.2 (Essential Partial Substitution). Given a PM-query $P Q$, an essential partial substitution $\theta$ for $P Q$ is an answer substitution for a partial PM-query of $P Q$.

Example 5.3. Consider the PM-queries of our running example, ignoring edge annotations for now. Observe that $? c 1 \leftarrow ? b 1 \& ? c 2 \leftarrow ? b 1 \leftarrow \mathrm{B} 1$ is a partial PM-query for $P Q_{1}$, and $? c 1 \leftarrow ? b 1 \rightarrow ? c 2$ $\& \mathrm{C} 8 \rightarrow$ ?c $1 \leftarrow$ ?c $2 \leftarrow \mathrm{C} 7$ is a partial $\mathrm{PM}$-query for $P Q_{2}$. The essential partial substitutions obtained in this case for $P Q_{1}$ and $P Q_{2}$ are reported in Table 3. Now, each vertex onto which a substitution maps variable ? $b 1$ has a chance to be in the top- $k$ answer. This chance may depend on the number of

\footnotetext{
${ }^{4}$ It should be observed that these paths may include a subset of the non-essential variables in $P Q$.
} 
Table 4. Candidate Output Table for $P Q_{1}$ and $P Q_{2}$ (without Annotations)

\begin{tabular}{|c|c|c|}
\hline Candidate output & Substitutions & Scoring term values \\
\hline \hline B2 & $\theta_{1,3}, \theta_{1,4}, \theta_{2,1}, \theta_{2,2}, \theta_{2,5}$ & $5,2,4,5,4$ \\
\hline B3 & $\theta_{1,1}, \theta_{1,2}, \theta_{2,4}$ & $2,2,4$ \\
\hline B4 & $\theta_{2,3}$ & 4 \\
\hline
\end{tabular}

full substitutions for the whole $P M$-queries $P Q_{1}$ and $P Q_{2}$ to which an essential partial substitution can be extended after mapping variables that do not belong to the partial PM-queries.

\subsection{Computing Upper Bounds on Scores}

Let us assume for now that, given an S-query $S Q=\langle P Q S, g a\rangle$, we have chosen a partial PM-query for each $P Q_{i} \in P Q S$ and we also have at hand all the possible essential partial substitutions for each of the partial PM-queries chosen.

We use these essential partial substitutions to compute upper bounds on local and global scores by creating a candidate output table, where for each candidate output vertex $v$, we maintain a list of essential partial substitutions that map an output variable to $v$, along with the values of the scoring terms obtained from the application of such substitutions. For instance, in the case of our running example, we create the candidate output table reported in Table 4.

Now, given a PM-query $P Q_{i} \in P Q S$ with $l a \in\{M I N, M A X, A V G\}$, we observe that an upper bound on $l s c o r e\left(v, P Q_{i}\right)$ is the maximum among the scoring term values of $v$ provided by all essential partial substitutions for $P Q_{i}$ that map an output variable to $v$.

If $l a=S U M$, it does not suffice to just include the scoring term value associated with a vertex $v$ by an essential partial substitution $\theta$ for $P Q_{i}$ in the computation of an upper bound on lscore $\left(v, P Q_{i}\right)$. Assume $\theta$ is computed for a partial PM-query $P P Q_{i}$ of $P Q_{i}$. The scoring term value provided by $\theta$ must be multiplied by an upper bound on the number of substitutions $\theta$ can be extended to after mapping the variables in $P Q_{i}$ that were not chosen as variables of $P P Q_{i}$. We compute this upper bound as $s u b^{*}\left(P Q_{i} \theta\right)$, where $s u b^{*}$ is a function that, given any PM-query $P Q,{ }^{5}$ returns an upper bound on the number of answer substitutions for the query.

It should be observed that, although conceptually $A V G$ is merely equal to $S U M$ over the number of matches, in the case of $A V G$ we still just take the maximum of the scoring term values as we do for $M I N$ and $M A X$. The following example explains why this is necessary.

Example 5.4. Consider the case where we have computed a set $\left\{\theta_{1}, \ldots, \theta_{n}\right\}$ of essential partial substitutions and their associated scoring term values are $x_{1}, \ldots, x_{n}$ with $x_{i} \leq x_{n}$ for any $i \in$ [1..n]. In this case, the correct upper bound under $A V G$ is the maximum value $x_{n}$, because we must take into account the "extreme" case where $\theta_{n}$ is the only partial substitution that will eventually be extended to a full one. Including any $x_{i}<x_{n}$ in the computation would provide a lower value, thus an incorrect upper bound.

The above example also provides an intuition about why the proposed framework could be seamlessly extended to handle, e.g., MODE and MEDIAN (they can be treated the same way as $M I N, M A X$, and $A V G$ ) and COUNT (which can be reduced to $S U M$ by fixing scores to 1 ). In general, the framework immediately supports any aggregation function $F$ such that, given a set $X$ of values, it always holds that $M I N(X) \leq F(X) \leq M A X(X)$ (we call this family of functions "range-closed" in the remainder).

\footnotetext{
${ }^{5}$ Observe that $P Q_{i} \theta$ is a PM-query itself.
} 
Table 5. Example Values of Function cand,

Where $\stackrel{\ell}{\leftarrow}$ Stands for $(\ell$, in $)$ and $\stackrel{\ell}{\rightarrow}$ for $(\ell$, out $)$

\begin{tabular}{|c|c|c|c|c|}
\hline$v$ & $\delta$ & $\pi$ & $e$ & cand \\
\hline B1 & 1 & bank & partner & 2 \\
\hline & ? & & funded & \\
\hline BI & Z & company & nart & 3 \\
\hline $\mathrm{C} 2$ & 1 & company & Partiner & 3 \\
\hline $\mathrm{C} 2$ & 2 & bank & $\stackrel{\text { partner }}{\longleftarrow}$ & 1 \\
\hline
\end{tabular}

Finally, an upper bound on $\operatorname{score}(v, S Q)$, denoted $\operatorname{score} e^{*}(v)$, is obtained by applying the same reasoning to the aggregation of the local scores of $v$ through function $g a$-the latter must therefore be range-closed as well.

Computing Upper Bounds on Substitutions. To quickly compute $s u b^{*}(P Q)$, we employ a structure called PM_Index. This index provides a value $\operatorname{cand}(v, \delta, \pi, e)$ for each vertex $v \in \mathcal{G}$, distance $\delta$, value $\pi \in \operatorname{dom}$ (type), and pair $e=(\ell, d)$, where $\ell \in \mathcal{L}$ and $d \in\{$ in, out $\}$. This value is computed as follows:

$-\operatorname{cand}(v, 1, \pi, e)$ is the cardinality of the set of vertices $v^{\prime}$ such that (i) $v^{\prime} . t y p e=\pi$ and (ii) $\mathcal{G}$ contains an edge $\left(v, l, v^{\prime}\right)$ if $d=$ out, an edge $\left(v^{\prime}, l, v\right)$ otherwise.

$-\operatorname{cand}(v, \delta, \pi, e)=\max \{\operatorname{cand}(u, 1, \pi, e) \mid$ there is an undirected path of length $\delta-1$ from $v$ to $u$ in $\mathcal{G}$.

Intuitively, for a given $(v, 1, \pi, e)$ quadruple (i.e., distance 1$)$, the index contains, for each label $\ell$, the number of in- and out-neighbors of $v$ that are connected via an edge labeled $\ell$ that are of type $\pi$. The second part of the definition updates this for the case when $\delta>1$ by recursively building on top of smaller values of $\delta .{ }^{6}$ Some values of function cand for the graph database of our running example are shown in Table 5.

Now, for each variable $? x$ in $P Q$, we can define an upper bound on the number of substitutions for $? x$ as $\operatorname{cand}^{*}(P Q, ? x)=\operatorname{cand}(v, \delta, \pi, e)$, where $v$ is a constant vertex of $P Q$ whose distance $\delta$ from ? $x$ is minimum w.r.t. the other constant vertices of $P Q, \pi$ is the value of the type property of $? x$, and the last edge of a shortest path between $v$ and $? x$ is $(\cdot, \ell, ? x)$ if $e=(\ell$, out $)$, and $(? x, \ell, \cdot)$ otherwise. $^{7}$

Finally, we can compute $s u b^{*}(P Q)$ by multiplying the values of $\operatorname{cand}^{*}(P Q, ? x)$ for all variables $? x$ in $P Q$. The following theorem ensures the correctness of this computation. The proofs for all the theorems in this article can be found in the Appendix.

Theorem 5.5. Suppose $P Q=\left\langle V_{P Q}, E_{P Q}, \chi, \varrho, l a\right\rangle$ is a PM-query. Then,

$$
\operatorname{sub}^{*}(P Q)=\prod_{? x \in V_{P Q} \cap V A R} \operatorname{cand}^{*}(P Q, ? x)
$$

is an upper bound on the number of substitutions for $P Q$.

\footnotetext{
${ }^{6}$ In the worst case, the construction of the PM_Index takes $O\left(|V|^{2}\right)$ time and its size is $O(|V|)$. However, our experiments on real datasets will show that actual construction times and index sizes are satisfactory in practice.

${ }^{7}$ We chose to use the shortest path in the definition of $\operatorname{cand}(P Q, ? x)$ for simplicity. We could have used any other kind of path in the definition-Theorem 5.5 can easily be extended to hold anyway.
} 
Example 5.6. In our running example, we have $\operatorname{sub}^{*}\left(P Q_{2}\right)=\prod_{? V \in\{? b 1, ? c 1, ? c 2, ? p 1, ? p 2\}}$ cand $^{*}\left(P Q_{2}\right.$, $? V)=12$ because $\operatorname{cand}^{*}\left(P Q_{2}, ? c 1\right)=\operatorname{cand}(C 8,1, \operatorname{company}, \stackrel{\text { partner }}{\longrightarrow})=3, \operatorname{cand}^{*}\left(P Q_{2}, ? c 2\right)=$ $\operatorname{cand}(C 7,1, \quad$ company, $\stackrel{\text { partner }}{\longrightarrow})=2, \quad$ and $\operatorname{cand}^{*}\left(P Q_{2}, ? b 1\right)=\operatorname{cand}(C 7,2$, bank, $\stackrel{\text { funded }}{\longleftarrow})=2$, $\operatorname{cand}^{*}\left(P Q_{2}, ? p 1\right)=\operatorname{cand}(C 8,2$, person, $\stackrel{\text { work }}{\longleftarrow})=1, \operatorname{cand}^{*}\left(P Q_{2}, ? p 2\right)=\operatorname{cand}(C 7,2$, person, $\stackrel{\text { work }}{\longleftarrow})=$ 1. Note that $C 7$ is the closest constant from ?c2, ?p2 and ?b1 while $C 8$ is the closest one from ?c1 and $? p 1$.

Later, our experiments will show that this way of computing upper bounds provides reasonably good bounds in practice.

\subsection{Choosing Partial PM-queries}

Given a PM-query $P Q$, the problem of choosing a partial PM-query $P P Q=\operatorname{opt}(P Q)$ for it is that of ensuring that mapping the variables of $P P Q$ against $G$ results in maximizing sub ${ }^{*}(P Q \theta)$ where $\theta$ is an answer substitution for $P P Q$. In other words, we want to define function opt in such a way that $\operatorname{sub}^{*}(P P Q, P Q)=\prod_{? x \in\left(V_{P Q} \backslash V_{P P Q}\right) \cap V A R} c a n d^{*}(P Q, ? x)$, which is the upper bound on the number of substitutions for the variables in $P Q$ that were not chosen as variables of $P P Q$, is maximized. ${ }^{8}$

We first show that the problem of computing opt $(P Q)$ is NP-hard, then we propose two approaches for addressing it.

To show NP-hardness of opt $(P Q)$, we start by introducing a decisional variant $\operatorname{opt}(P Q)_{\text {log }}^{B}$ defined as the problem of deciding whether there is a partial PM-query $P P Q$ for $P Q$ such that $\log _{2} s u b^{*}(P P Q, P Q) \geq B .{ }^{9}$ Theorem 5.7 characterizes the complexity of this variant.

Theorem 5.7. opt $(P Q)_{\log }^{B}$ is NP-complete.

Now, it is easy to observe that $\operatorname{opt}(P Q)_{\log }^{B}$ can be polynomially decided after solving the original problem opt $(P Q)$. Indeed, opt $(P Q)_{\text {log }}^{B}$ is true iff opt $(P Q)$ returns a partial PM-query $P P Q$ such that $\log _{2} s u b^{*}(P P Q, P Q) \geq B$. Thus, opt $(P Q)$ is NP-hard.

To compute opt $(P Q)$ we propose two alternative approaches: a list-oriented pruning method and a reduction to the NWST problem.

List-Oriented Pruning. Given a PM-query $P Q$, a partial PM-query for $P Q$ can be generated by choosing a set $V_{s} \subset V_{P Q}$, and then checking whether Definition 5.1 holds for the partial PM-query induced by the vertices in $V_{s}$ (denoted $P Q\left(V_{s}\right)$ ). The list-oriented pruning method starts by building the list of all subsets $V_{s}$ of $V_{P Q}$ s.t. $\left|V_{s}\right|=\left|V_{P Q}\right|-1$. Then, the list is processed as follows. For each unprocessed subset $V_{s}$ in the list, if $P Q\left(V_{s}\right)$ satisfies Definition 5.1, then the algorithm adds all the subsets of $V_{s}$ with cardinality $\left|V_{s}\right|-1$ to the list-otherwise, $V_{s}$ is immediately pruned from the list. When all the subsets in the list have been processed, the algorithm selects the one for which $s u b^{*}\left(P Q\left(V_{s}\right), P Q\right)$ is maximum. It is easy to see that if $V_{s}$ does not comply with Definition 5.1, then no subset of $V_{s}$ can do: (i) if $\mathcal{E}(P Q) \nsubseteq V_{s}$, then we have $\mathcal{E}(P Q) \nsubseteq V_{s}^{\prime}$ for any $V_{s}^{\prime} \subset V_{s}$; (ii) if a path between the essential variables of $P Q$, or between an essential variable and a constant vertex, cannot be defined using the vertices in $V_{s}$, then no path can be defined using any subset of $V_{s}$.

\footnotetext{
${ }^{8}$ The reason why we want to maximize $s u b^{*}(P P Q, P Q)$ is related to how pruning is applied. Intuitively, during query processing, if we pick the vertices that will possibly get a higher exact score, we increase the chances that more vertices will drop below the current threshold. Further details are provided in Section 5.4.

${ }^{9}$ For technical reasons, we use log. It allows us to consider $B$ such that its size is polynomial w.r.t. $\left|V_{P Q}\right|$ in the reduction of Theorem 5.7 .
} 
For instance, if we consider $P Q_{2}$ of our running example, $V_{s}=\{\mathrm{C} 7, ? b 1, ? c 2, ? p 2\}$ does not comply with Definition 5.1 because ? $c 1$ is a scoring variable, so the algorithm avoids listing all of its subsets.

Reduction to the NWST Problem. The second approach reduces our problem to the NWST problem and then uses well-known approximate algorithms for NWST to compute (sub)optimal partial PM-queries. The NWST problem is defined as follows. Given an undirected graph $\widehat{G}=\langle\widehat{V}, \widehat{E}\rangle$, a node-weight function $\omega: \widehat{V} \rightarrow \mathbb{N}$, and a subset of vertices $S \subseteq \widehat{V}$ (called terminals), compute the minimum weight subtree of $\widehat{G}$ that includes all the vertices in $S$.

We reduce the problem of computing opt $(P Q)$ to an instance $N W S T(P Q)$ of NWST as defined below.

Definition 5.8 (NWST(PQ)). Given a PM-query $P Q=\left\langle V_{P Q}, E_{P Q}, \chi, \varrho, l a\right\rangle$, an instance $\operatorname{NWST}(P Q)$ of NWST is defined as follows: (i) $\widehat{V}$ contains the same vertices as $P Q$; (ii) $\widehat{E}$ contains an undirected edge $\left(v_{1}, v_{2}\right)$ for each edge $\left(v_{1},\{\ell\}, 1, v_{2}\right)$ or $\left(v_{2},\{\ell\}, 1, v_{1}\right)$ in $E_{P Q}$; (iii) $S=\mathcal{E}(P Q) \cup\left(V_{P Q} \cap V\right)$, i.e., $S$ consists of all the essential variables and constant vertices of $P Q$; (iv) $\forall v \in S, \omega(v)=0$ and $\forall ? x \in \widehat{V} \backslash S, \omega(v)=\ln \operatorname{cand}^{*}(P Q, ? x)$.

It turns out that solving $N W S T(P Q)$ suffices to solve opt $(P Q)$.

Theorem 5.9. Given a PM-query $P Q$, every solution $T$ of NWST $(P Q)$ one-to-one corresponds to a partial $P M$-query $P P Q(T)$ for $P Q$ such that sub ${ }^{*}(P P Q(T), P Q)$ is maximum.

Thus, many well-known approximate algorithms for NWST can be used to compute opt $(P Q)$. For instance, Reference [21] presents two algorithms with $1.35 \ln |S|$ and $1.65 \ln |S|$ as the worstcase approximation ratios. Interestingly, these approximation ratios do not depend on the size of the graph but only on the number of terminal vertices, which corresponds in our case to the number of constant vertices and essential variables. In our implementation, we used the faster $1.65 \ln |S|$ algorithm.

\subsection{The PScore Algorithm}

We conclude the section by showing how the various techniques described in the previous sections fit together in our overall query-processing algorithm, named PScore (Algorithm 1).

The algorithm, on Line 5, applies one of the two optimization methods described in Section 5.3. If the list-oriented pruning method is applied, we call the algorithm PScore_LP; otherwise, we call it PScore_NWST.

The computation of exact scores (Lines 12 and 20) is done by trying to extend every partial substitution $\theta$ associated with a vertex in $T$ to a "full" substitution (i.e., by computing an answer substitution for $P Q_{i} \theta$ if $\theta$ is an answer substitution for $\left.P P Q_{i}\right)$. To achieve this, the algorithm employs the map algorithm described in Section 6.2.

It should be observed that:

- The algorithm prunes away all vertices in the table whose upper bound is less than or equal to the exact score of the $k$-th result, by comparing this score with the maximum upper bound of the unprocessed vertices (Lines 15-19).

- The algorithm gracefully handles negative scores and anti-monotonic range-closed functions thanks to the use of essential partial substitutions. In fact, the upper bounds computed for the vertices in $T$ are independent of whether scoring terms may produce negative scores or aggregation functions are anti-monotonic-upper bounds are "automatically" kept correct by the fact that range-closed aggregation functions are guaranteed to keep their values in the expected range. 


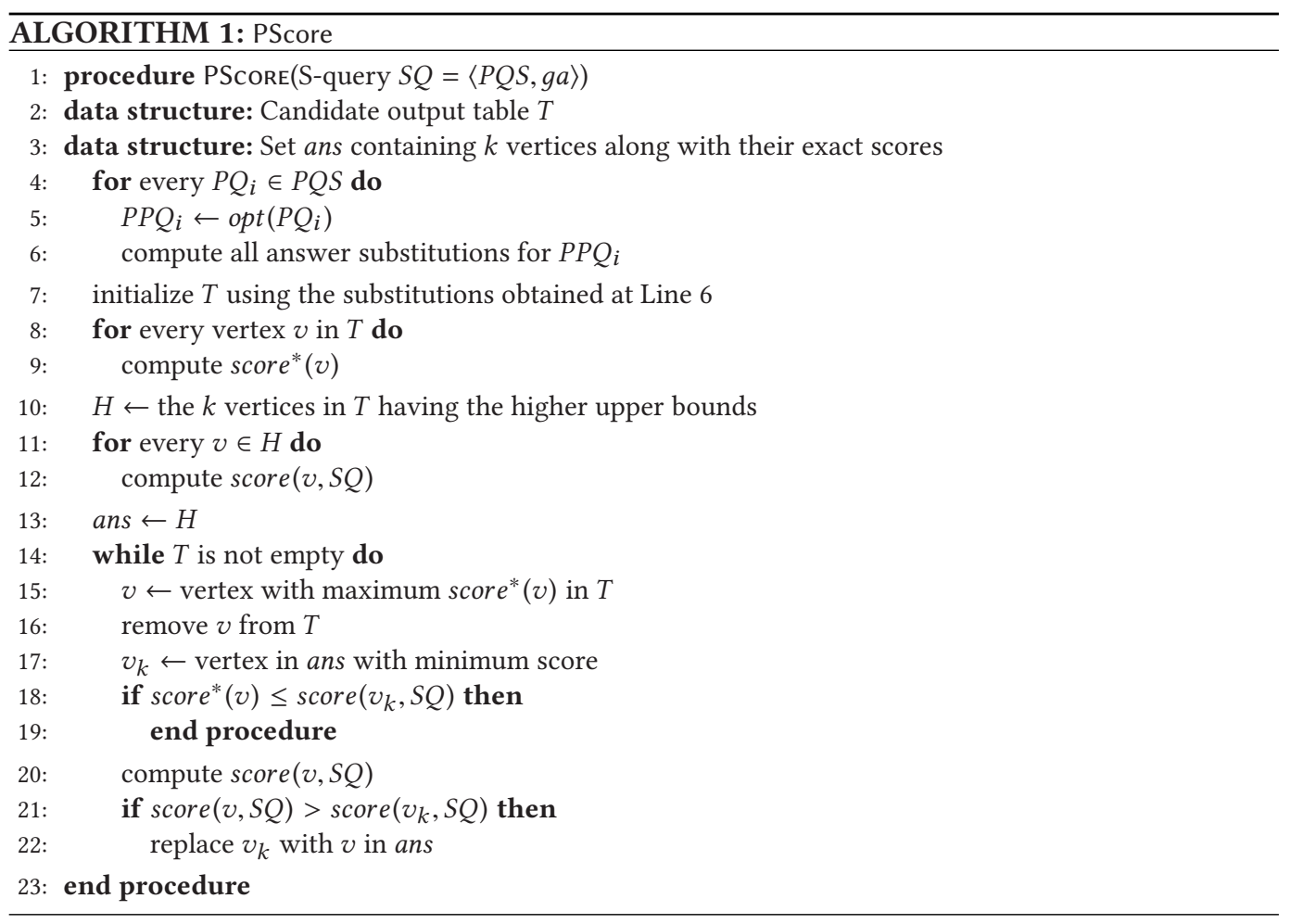

- The algorithm proceeds in decreasing order of upper bounds because of the way vertices in the table are pruned. If the condition on Line 21 is true, then a vertex $v$ has an exact score that makes it part of the current top- $k$ answer. When this happens, the upper bounds of one or more vertices in the table drop below the current threshold-such vertices are "automatically" pruned as explained above. It can easily be observed that the higher the score of $v$, the higher the likelihood that a larger set of vertices will be pruned.

Proposition 5.10. Given an S-query $S Q=\langle P Q S, g a\rangle$, the PScore algorithm terminates and correctly computes ans $(S Q)$. Its worst-case asymptotical time complexity is $O\left(T_{\text {map }} \cdot|V|^{M}\right)$ where $M=$ $\max _{P Q \in P Q S}\left|V_{P Q} \cap V A R\right|$ and $T_{\text {map }}$ is the complexity of the map algorithm.

Finally, it should also be observed that the PScore algorithm assumes the existence of at least $k$ full substitutions-this is because Definition 4.6 requires $|\operatorname{ans}(S Q)|=k$. However, the algorithm can be easily adapted to the case where the number of substitutions is lower than $k$ and thus no top- $k$ answer must be returned. In fact, it suffices to check that $T$ (resp., ans) contains at least $k$ vertices along with their scores after Line 7 (resp., Line 13) and end the procedure otherwise.

\section{MANAGING EDGE-ANNOTATED QUERIES}

In this section, we discuss how we extend the techniques presented in Section 5 to handle the case of edge-annotated PM-queries. In particular, Section 6.1 shows our approach to the computation of upper bounds and Section 6.2 describes the algorithm for computing substitutions. 


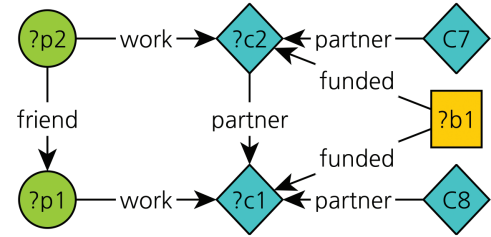

(a) $E Q_{1}$

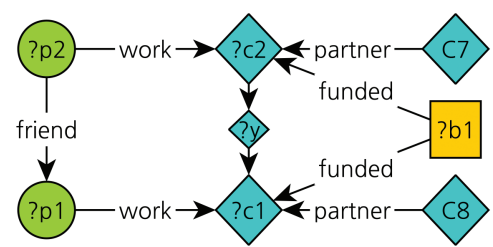

(c) $E Q_{3}$

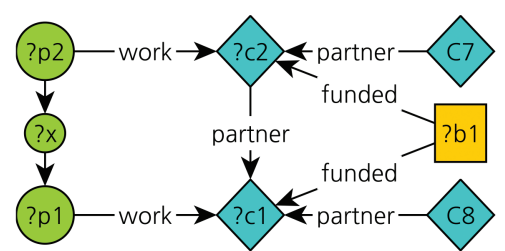

(b) $E Q_{2}$

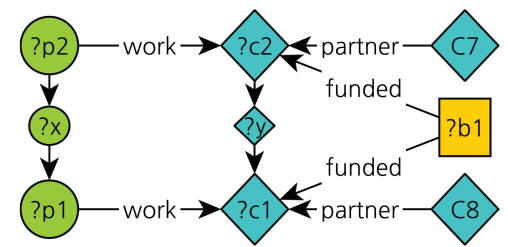

(d) $E Q_{4}$

Fig. 4. Expanded queries for $\mathrm{PQ}_{2}$ of Figure 1.

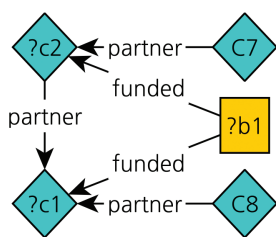

(a) $P P Q_{2,1}$

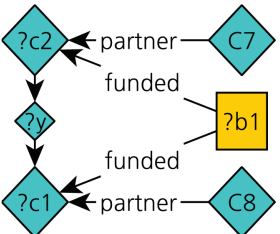

(b) $P P Q_{2,2}$

Fig. 5. Two partial PM-queries for $P Q_{2}$.

\subsection{Computing Upper Bounds on Scores}

The computation of upper bounds for edge-annotated queries can take advantage of specific relationships among partial substitutions that are present in this case.

Consider for instance $P Q_{2}$ of our running example. An upper bound for it could be computed by (1) expanding it into an equivalent set eq $\left(P Q_{2}\right)=\left\{E Q_{1}, E Q_{2}, E Q_{3}, E Q_{4}\right\}$ (shown in Figure 4) of non-annotated "expanded" PM-queries for $P Q$, (2) processing such expanded queries one-by-one, and then (3) taking the sum of the resulting upper bounds (since the set of substitutions for $P Q_{2}$ is the union of the sets of substitutions for the expanded queries).

However, this would require processing a partial PM-query for each expanded query, and the number of expanded queries grows very quickly. Consider an edge-annotated PM-query $P Q=$ $\left\langle V_{P Q}, E_{P Q}, \chi, \varrho, l a\right\rangle$. For each annotated edge $e_{i}=\left(v_{i}, L_{i}, w_{i}, v_{i}^{\prime}\right)$ in $E_{P Q}$, let $\exp \left(e_{i}\right)$ be the set of edges $\left.\left\{\left(v_{i}, L_{i}, 1, v_{i}^{\prime}\right),\left(v_{i}, L_{i}, 2, v_{i}^{\prime}\right)\right\}, \ldots,\left(v_{i}, L_{i}, w_{i}, v_{i}^{\prime}\right)\right\}$. Now, observe that each expanded query in $e q(P Q)$ contains a different set of edges from $\exp \left(e_{1}\right) \times \cdots \times \exp \left(e_{n}\right)$ where $n$ is the number of annotated edges in $E_{P Q}$. Thus, there are $\prod_{i \in[1 . . n]} w_{i}$ expanded queries for $P Q$.

We therefore propose a different approach that heuristically chooses a set of partial PM-queries such that by extending a substitution for one of them, we can obtain full substitutions for more than one expanded query.

Example 6.1. By extending an essential partial substitution for $P P Q_{2,1}$ in Figure 5 (resp., $P P Q_{2,2}$ ), full substitutions for $E Q_{1}$ and $E Q_{2}$ (resp., $E Q_{3}$ and $E Q_{4}$ ) can be built. Now, suppose we compute a 
substitution $\theta_{1}$ for $P P Q_{2,1}$. This substitution will not map variables ?p1 and ?p2 of $E Q_{1}$ and variables ?p1, ?p2, and ?x of $E Q_{2}$. The upper bound on the number of substitutions obtainable by extending $\theta_{1}$ is therefore $s u b^{*}\left(E Q_{1} \theta_{1}\right)+s u b^{*}\left(E Q_{2} \theta_{1}\right)=\operatorname{cand}^{*}\left(E Q_{1} \theta_{1}, ? p 1\right) \times \operatorname{cand}^{*}\left(E Q_{1} \theta_{1}, ? p 2\right)+$ $\operatorname{cand}^{*}\left(E Q_{2} \theta_{1}, ? p 1\right) \times \operatorname{cand}^{*}\left(E Q_{2} \theta_{1}, ? p 2\right) \times \operatorname{cand}^{*}\left(E Q_{2} \theta_{1}, ? x\right)$. The same applies to any substitution $\theta_{2}$ for $P P Q_{2,2}$ : its upper bound is $\operatorname{sub}^{*}\left(E Q_{3} \theta_{2}\right)+\operatorname{sub}\left(E Q_{4} \theta_{2}\right)=\operatorname{cand}^{*}\left(E Q_{3} \theta_{2}, ? p 1\right) \times$ $\operatorname{cand}^{*}\left(E Q_{3} \theta_{2}, ? p 2\right)+\operatorname{cand}^{*}\left(E Q_{4} \theta_{2}, ? p 1\right) \times \operatorname{cand}^{*}\left(E Q_{3} \theta_{2}, ? p 2\right) \times \operatorname{cand}^{*}\left(E Q_{4} \theta_{2}, ? x\right)$.

In general, let $P Q$ be a PM-query and $P P Q$ a partial PM-query for it. Moreover, let $\operatorname{cov}(P P Q) \subseteq$ $e q(P Q)$ be the set of expanded queries such that by extending a substitution for $P P Q$, we obtain full substitutions for all the queries in $\operatorname{cov}(P P Q)$-for instance, in our example we have $\operatorname{cov}\left(P P Q_{2,1}\right)=$ $\left\{E Q_{1}, E Q_{2}\right\}$. Given a substitution $\theta$ for $P P Q$, we have $s u b^{*}(P Q \theta)=\sum_{E Q \in \operatorname{cov}(P P Q)} \operatorname{sub}(E Q \theta)$.

We now describe our heuristics to choose a set $Q$ of partial PM-queries for an edge-annotated PM-query $P Q$. Obviously, $Q$ must "fully cover" the set of expanded queries for $P Q$, that is, it must satisfy $\cup_{P P Q \in Q} \operatorname{cov}(P P Q)=e q(P Q)$. To ensure this, and to possibly provide high-quality partial $P M-q u e r i e s$, we first build a modified version $P Q^{\prime}$ of $P Q$ (by replacing annotated edges with nonannotated ones), then we compute opt $\left(P Q^{\prime}\right)$ and use the result to compute $Q$.

Set $E_{P Q^{\prime}}$ is built from $E_{P Q}$ by replacing each annotated edge $e=\left(v, L, w, v^{\prime}\right)$ with two edges $\bmod _{1}(e)=(v, L, 1, ? V)$ and $\bmod _{2}(e)=\left(? V, L, 1, v^{\prime}\right)$ with $? V$ being an artificial variable not in $V_{P Q}-$ the number of such new edges is $2 n$, i.e., twice the number of annotated edges in $E_{P Q}$. Now, to compute $P P Q^{\prime}=\operatorname{opt}\left(P Q^{\prime}\right)$ we need to choose a good value for $c a n d^{*}\left(P Q^{\prime}, ? V\right)$ - this value cannot be derived from the PM_Index as ? $V$ is an artificial variable we introduce just for dealing with annotated edges. Consider the "longest expansion" of $e$ that consists of the following path of $w$ non-annotated edges: $\left(v, L, 1, ? V_{1}\right),\left(? V_{1}, L, 1, ? V_{2}\right), \ldots,\left(? V_{w-1}, L, 1, v^{\prime}\right)$ with each $? V_{i}$ being an artificial variable not in $V_{P Q}$. We define cand $^{*}\left(P Q^{\prime}, ? V\right)$ as $\frac{1}{\left|e q\left(P Q^{\prime}\right)\right|} \sum_{i \in[1 . . w]} \sum_{j \in\left[1 . .\left|e q\left(P Q^{\prime}\right)\right|\right]} c_{a n d}\left(E Q_{j}, ? V_{i}\right)$. Observe that due to the properties of the problem of choosing optimal partial PM-queries, function opt will either include both edges $\bmod _{1}(e)$ and $\bmod _{2}(e)$ in $P P Q^{\prime}$, or none of them. The final set $Q$ can then be computed from $P P Q^{\prime}$ as follows. For each annotated edge $e_{i}=\left(v_{i}, L_{i}, w_{i}, v_{i}^{\prime}\right)$ in $E_{P Q}$, we define a set of edges $E_{Q}\left(e_{i}\right)$ as $\exp \left(e_{i}\right)$ if $\bmod _{1}\left(e_{i}\right)$ is in $P P Q^{\prime}$, and $\emptyset$ otherwise. Now we consider the set $E C=E_{Q}\left(e_{1}\right) \times \cdot \times E_{Q}\left(e_{n}\right)$. Set $Q$ is initialized with $|E C|$ partial PM-queries, containing only the non-annotated edges in $E_{P Q}$. Each of these queries is then extended with a different set of edges from $E C$. Observe that $|Q|=|E C|=\prod_{i \in[1 . . n], \bmod _{1}\left(e_{i}\right) \text { in } P P Q^{\prime}} w_{i}$. It is easy to see that this approach ensures that $Q$ covers the set of expanded queries for $P Q$.

For instance, in our running example, if opt $\left(P Q_{2}\right)$ is a partial PM-query of the form $? c 1 \leftarrow ? b 1 \rightarrow$ $? c 2 \& \mathrm{C} 8 \rightarrow ? \mathrm{c} 1 \leftarrow ? \mathrm{c} 2 \leftarrow \mathrm{C} 7$, then the final partial PM-queries are those reported in Figure 5.

As our experiments will show, this approach chooses good-quality partial PM-queries. Moreover, the structure of the map algorithm presented in Section 6.2 allows us to exploit commonalities between the queries in $Q$. In fact, the actual set of queries map must run over can just include the edges with the maximum-length annotation (i.e., $\left(v_{i}, L_{i}, w_{i}, v_{i}^{\prime}\right)$ in set $E_{Q}\left(e_{i}\right)$ above). The number of such queries is $\mid\left\{e_{i}\right.$ in $P Q^{\prime} \mid \bmod _{1}\left(e_{i}\right)$ in $\left.P P Q^{\prime}\right\} \mid$. In our running example, the input to the map algorithm would be the single partial PM-query ?c1 $\leftarrow$ ? $b 1 \rightarrow ? c 2 \& \mathrm{C} 8 \rightarrow$ ?c $1 \stackrel{2}{\leftarrow}$ ?c $2 \leftarrow \mathrm{C} 7$.

\subsection{Mapping Algorithm}

Algorithm 2 computes all answer substitutions for a given (edge-annotated) PM-query $P Q$ against the graph database $\mathcal{G}$.

The algorithm essentially performs a depth-first search and, when it finds an edge $e$ with an annotation greater than 1 (denoted $\operatorname{ann}(e)>1$ ) it starts a separate depth-first search using a copy of the query obtained by decreasing the annotation by 1 . In the algorithm, $S$ is the set of substitutions 
being computed. Moreover, the algorithm uses an external function mcand $(u, L, ? v)$ that returns the set of vertices of $\mathcal{G}$ that can be matched to ? $v$, i.e., those that are directly connected to $u$ through an edge with a label in $L$. Note that $\mid$ mcand $(u, L, ? v) \mid$ can be retrieved from the PM_Index. Access to graph data on disk is managed through the DOGMA index [7].

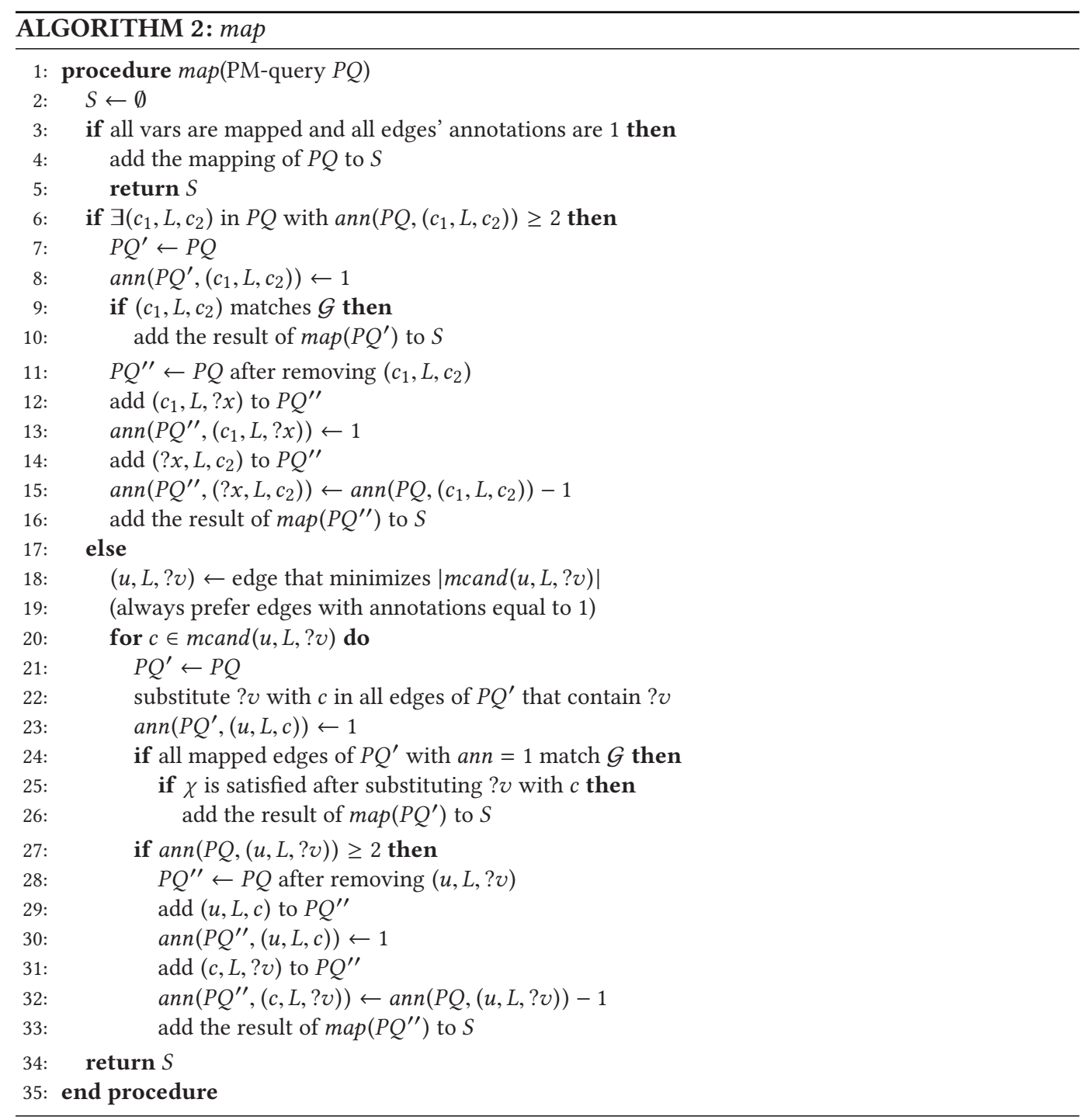

In the algorithm, Lines 6-16 deal with the case of fully mapped edges with an annotation greater than 1, whereas Lines 18-33 deal with unmapped edges. In the first case, given an edge $e$ to process, we create a copy $P Q^{\prime}$ of $P Q$ with ann $(e)=1$, and call map $\left(P Q^{\prime}\right)$ recursively to continue its mapping (we call this the "Branch" operation). We also create a $P Q^{\prime \prime}$ where $e$ is expanded to two edges whose annotations are 1 and $\operatorname{ann}(e)-1$, respectively, and call map $\left(P Q^{\prime \prime}\right)$ recursively ("Spawn" operation). In the second case, we have to select one unmapped edge to find substitutions for (Line 18). We select the edge $(u, L, ? v)$ for which the number of vertices that can be matched to ?v is minimized. 


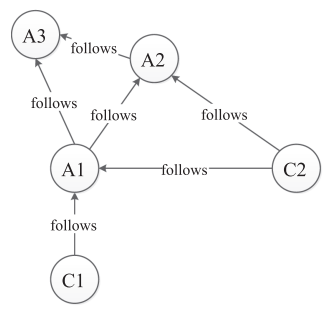

(a) Graph database

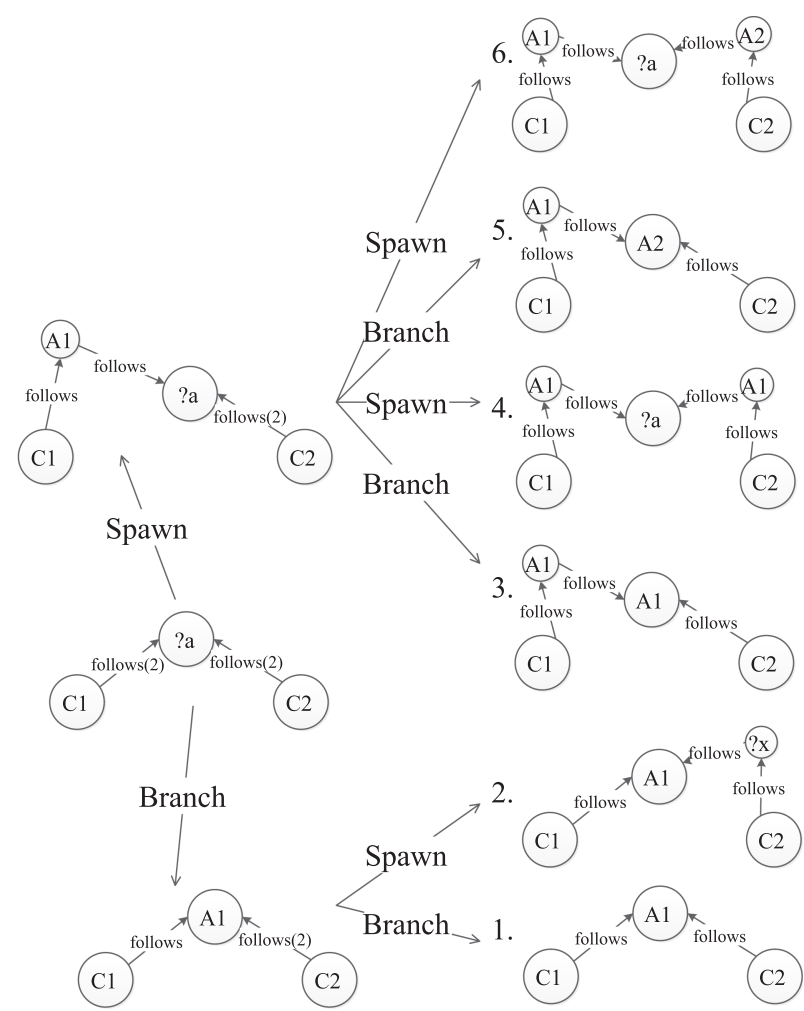

(b) Branch and spawn operations

Fig. 6. Example graph database (a) and execution of the map algorithm up to the second level of recursion (b).

Then, for each vertex $c \in \operatorname{mcand}(u, L, ? v)$, we create $P Q^{\prime}$ and $P Q^{\prime \prime}$ as we did in the first case, and call map recursively.

Subgraph isomorphism is checked on Lines 9 and 24. It should be noted that, until there is no mapped edge with annotation greater than 1, the processing of unmapped edges is deferred. We made this choice to maximize the chance to prune a branch on Line 9. It should also be observed that any SPARQL engine or subgraph matching algorithm could be used to check subgraph isomorphism-in our implementation we used the algorithm proposed for the DOGMA index [7].

Example 6.2. Consider the graph database in Figure 6(a) and the PM-query at the left of Figure 6(b). The algorithm starts by processing $C 1 \stackrel{\text { follows }(2)}{\longrightarrow}$ ? $a$. A1 is the only neighbor of $C 1$. For the branch operation, the algorithm substitutes ? $a$ with $A 1$. For the spawn operation, it expands the edge and substitutes the intermediate variable with $A 1$. Note that expanded edges have annotations equal to 1 and $\operatorname{ann}(C 1 \stackrel{\text { follows }(2)}{\longrightarrow} ? a)-1=1$, respectively. After branching, $C 2 \stackrel{\text { follows }(2)}{\longrightarrow} A 1$ is processed. In this case, for the branch operation the algorithm checks whether $C 2$ and $A 1$ are connected through follows in $\mathcal{G}$. If so, we have found an answer substitution (case 1 . in the 
Table 6. Graph Databases (Top) and Query Types (Bottom). In the Table, $n_{V}$ Is the Number of Vertices in Each PM-query $\left(\left|V_{P Q}\right|\right), n_{E}$ the Number of Edges $\left(\left|E_{P Q}\right|\right)$, and $n_{C}$ the Number of Constant Vertices $\left(\left|V_{P Q} \cap V\right|\right)$

\begin{tabular}{|c|c|c|c|c|c|}
\hline & $|V|$ & $|E|$ & & $|V|$ & $|E|$ \\
\hline \hline Nuclear [1] & $74 \mathrm{~K}$ & $1.1 \mathrm{M}$ & Flickr [9] & $6.2 \mathrm{M}$ & $15.2 \mathrm{M}$ \\
CiteSeerX [13] & $0.93 \mathrm{M}$ & $2.9 \mathrm{M}$ & BSBM [6] & $14.4 \mathrm{M}$ & $37.2 \mathrm{M}$ \\
IMDb [25] & $2.1 \mathrm{M}$ & $7.7 \mathrm{M}$ & Orkut [33] & $3.7 \mathrm{M}$ & $234 \mathrm{M}$ \\
YouTube [12] & $4.6 \mathrm{M}$ & $14.9 \mathrm{M}$ & & & \\
\hline
\end{tabular}

\begin{tabular}{|c|c|c|c|c|c|c|c|c|c|c|c|c|}
\hline & \multicolumn{3}{|c|}{ Small tree } & \multicolumn{3}{|c|}{ Small graph } & \multicolumn{3}{|c|}{ Large tree } & \multicolumn{3}{|c|}{ Large graph } \\
\cline { 2 - 12 } & $n_{V}$ & $n_{E}$ & $n_{C}$ & $n_{V}$ & $n_{E}$ & $n_{C}$ & $n_{V}$ & $n_{E}$ & $n_{C}$ & $n_{V}$ & $n_{E}$ & $n_{C}$ \\
\hline \hline Nuclear & 5 & 4 & 1 & 5 & 8 & 1 & 10 & 9 & 4 & 10 & 15 & 4 \\
CiteSeerX & 5 & 4 & 1 & 5 & 8 & 1 & 10 & 9 & 4 & 10 & 15 & 4 \\
IMDb & 5 & 4 & 2 & - & - & - & 9 & 8 & 4 & - & - & - \\
YouTube & 6 & 5 & 1 & 6 & 10 & 1 & 10 & 9 & 3 & 10 & 15 & 3 \\
Flickr & 5 & 4 & 1 & 5 & 6 & 1 & 10 & 9 & 4 & 10 & 11 & 4 \\
Orkut & 5 & 4 & 2 & 5 & 8 & 2 & 10 & 9 & 5 & 10 & 15 & 5 \\
\hline
\end{tabular}

figure). For the spawn operation, an intermediate variable $? x$ is added and the mapping continues. The process goes on until all substitutions (such as cases 1 and 5 in the figure) are found.

Proposition 6.3. Given a PM-query PQ, Algorithm 2 terminates and correctly computes all answer substitutions for $P Q$ in time $O\left(|e q(P Q)| \cdot|V|^{\left|V_{P Q} \cap V A R\right|+v}\right)$, where $v=\sum_{\left(v, S, w, v^{\prime}\right) \in E_{P Q}}(w-1)$.

\section{EXPERIMENTAL EVALUATION}

\subsection{Setting}

We tested our framework using the seven graph databases in Table 6. For all databases but BSBM, we generated $3 \mathrm{~K}$ S-queries, each containing three PM-queries. In turn, for each PM-query, we started by randomly retrieving a connected subgraph (or subtree) from the database with a fixed number of vertices and edges. We then replaced a fixed portion of the vertices with variables. We generated four different types of queries, whose numbers of vertices, edges, and constant vertices are reported in Table $6 .{ }^{10}$ For the IMDb database, which is mostly tree-structured, we only built tree-structured queries. For the BSBM dataset, we used a modified version of the six test queries in Reference [32], to which we added GROUP BY statements and anchors to create aggregate top- $k$ queries.

To vary the selectivity of the queries, we used a parameter $\gamma \in\{0.3,0.7\}$ that represents the percentage of variables with constraints (i.e., variables for which function $\varrho$ of Definition 4.1 is defined)-for a variable ? $v$ that replaced a vertex $u$ with property $u . p=z$, we wrote a constraint whose form is either ?v.p $\geq z$ or $? v \cdot p \leq z$ (if $u$ had multiple numerical properties, we chose one randomly). This way, we also guaranteed that there exists at least one substitution for each query (we actually obtained up to tens of millions of substitutions). Moreover, for each query, we

\footnotetext{
${ }^{10}$ For other works that use similar approaches to the selection of queries, see, e.g., References [6, 37, 39, 49].
} 
randomly chose a subset of variables and designated them as output variables (up to $50 \%$, with an average of 25\%). Functions $l a$ and $g a$ were chosen randomly as well.

We generated queries by starting from a group of $1 \mathrm{~K}$ S-queries containing only non-annotated PM-queries with single labels on all edges. We then generated $1 \mathrm{~K}$ annotated S-queries by randomly adding annotations to edges: $60 \%$ of edges had $w=1,25 \%$ had $w=2,10 \%$ had $w=3$, and $5 \%$ had $w=4 .{ }^{11}$ Finally, we generated $1 \mathrm{~K}$ annotated multiple-label S-queries by adding further labels on $40 \%$ of the edges.

We evaluated the performance of PScore_LP and PScore_NWST with different query types and values of $\gamma$. We compared our algorithms with (i) a PScore_Base algorithm that computes all answers to the query, scores them, and selects the top- $k,{ }^{12}$ and (ii) two of the most popular RDF query engines, Jena $[8,26,51]$ and GraphDB $[5,19]$ (formerly known as OWLim). We point out that none of these systems is specifically targeted at aggregate top- $k$ computation, whereas we apply a specialized approach to the problem-thus, we expected our algorithms to outperform such systems. Moreover, GraphDB strictly adheres to the SPARQL 1.1 standard, where path edges cannot have length constraints-thus, we tested GraphDB by first expanding each annotated PM-query $P Q$ into its equivalent set of non-annotated queries $e q(P Q)$ as described in Section 6.1. Finally, Jena supports path edges with limited path length as we do in our PM-queries, but it suffers from the limitations discussed in Section 2.

All the experiments were run on a Xeon $5140 \mathrm{CPU}$ clocked at $2.33 \mathrm{GHz}$, equipped with $16 \mathrm{~GB}$ RAM and running RedHat Linux.

\subsection{Results}

In a first round of experiments, we measured the query evaluation times obtained with $k=10$ using annotated single-label queries. The results are reported in Figure 7.

The evaluation times we obtained are very satisfactory in general. On all datasets but IMDb, the performance advantage of PScore_Base over GraphDB ranges between $35.8 \%$ and $75.8 \%$, with an average of $58.4 \%$. The only cases where PScore_Base and GraphDB show similar performance are those involving tree-structured queries on the IMDb dataset. Jena was not able to complete the query evaluation process in over half the cases (for each query, we fixed a timeout equal to 4 times the query evaluation time of PScore_Base) except for the BSBM dataset. The percentage of timeouts w.r.t. the total number of queries of a certain type/size/selectivity (see Table 7) was up to $90.5 \%$. Interestingly, the percentage of timeouts decreased around $20 \%$ on average when we used equivalent sets of non-annotated queries.

In addition, PScore_LP and PScore_NWST largely outperform PScore_Base on all datasets. The performance advantage ranges between $53.7 \%$ and $95.8 \%$, with an average of $75.8 \%$. This is mainly a consequence of the much smaller number of full substitutions PScore_LP and PScore_NWST need to compute. Figure 8 reports statistics about the average percentage of substitutions they processed w.r.t. PScore_Base during this round of experiments. The results show that both the algorithms save over $60 \%$ in the majority of cases, and over $90 \%$ in more than $30 \%$ of cases.

Generally, the relative performance of PScore_LP and PScore_NWST depends on the query type. In small tree queries, the quality of the partial PM-queries computed by PScore_NWST is very close to that obtained by PScore_LP, while requiring shorter computation times. In small graph queries, the advantage provided by PScore_LP's better partial PM-queries appears to

\footnotetext{
${ }^{11}$ We recall that $w$ is the integer value associated with annotated edges (Definition 4.1). Connections involving more than four edges have been shown to have very limited usefulness in practical scenarios [2].

${ }^{12}$ It should be observed that, although the PScore_Base algorithm does not apply any kind of pruning over the set of substitutions, it still benefits from the efficient disk access employed by the map algorithm.
} 
Nuclear
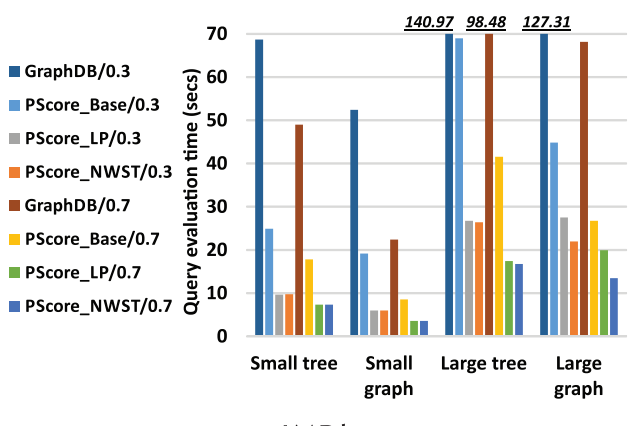

IMDb

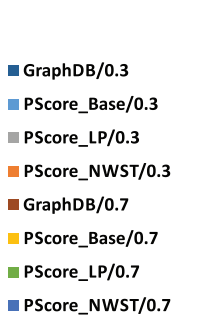

n PScore_NWST/0.7

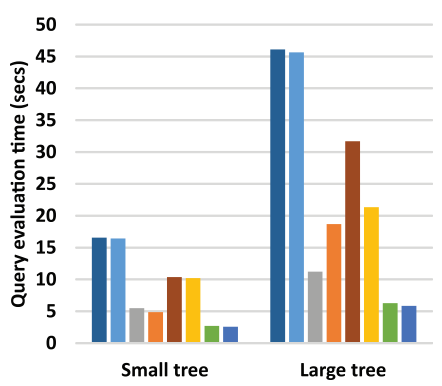

Flickr

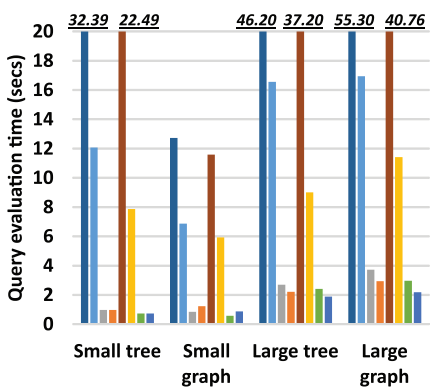

CiteSeerX

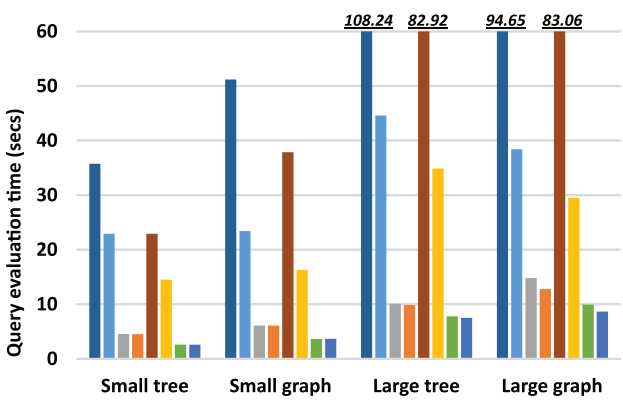

YouTube

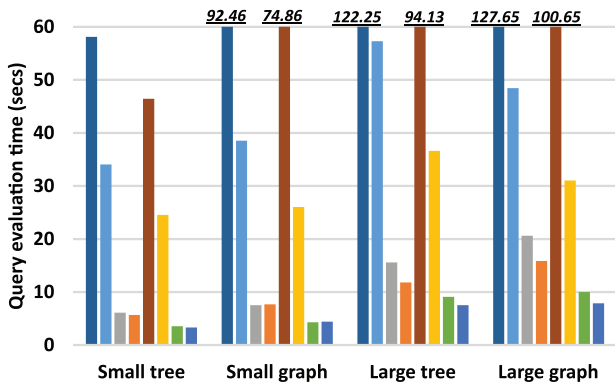

Orkut

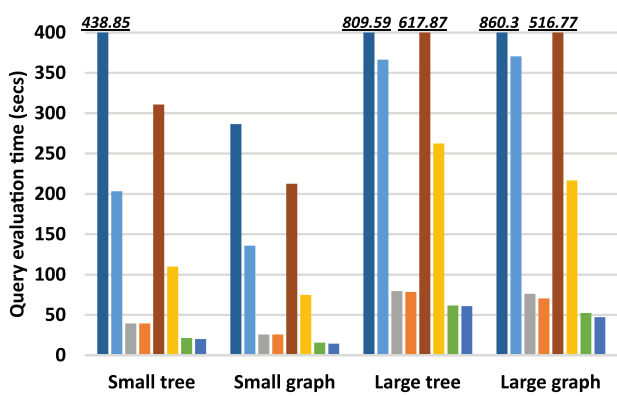

BSBM

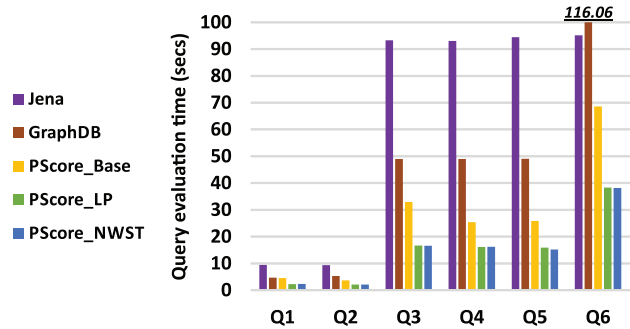

Fig. 7. Query evaluation time for different algorithms, values of $\gamma$, and query types-label $A / x$ means algorithm $A$ with $\gamma=x$. 
Table 7. Percentage of Jena Timeouts

\begin{tabular}{|c|c|c|c|c|}
\hline$\gamma$ & Small tree & Small graph & Large tree & Large graph \\
\hline 0.3 & $59.4 \%$ & $79.4 \%$ & $76.6 \%$ & $88 \%$ \\
0.7 & $61.4 \%$ & $81 \%$ & $83.7 \%$ & $90.5 \%$ \\
\hline
\end{tabular}

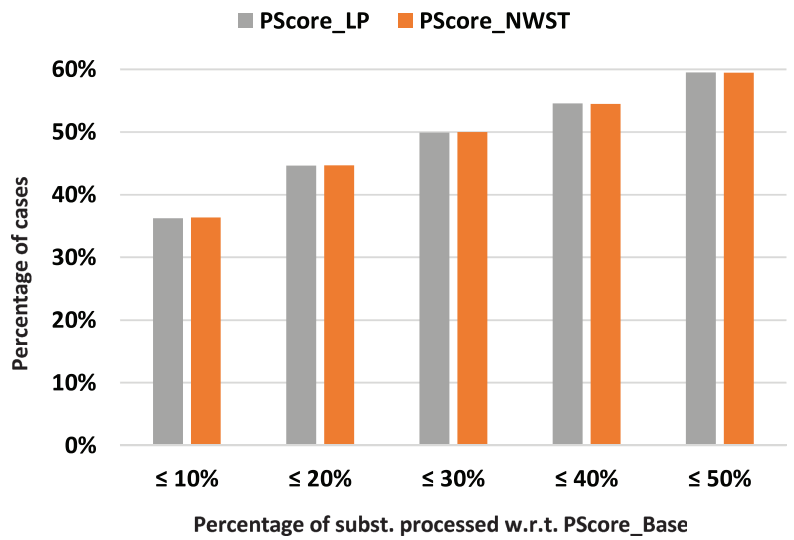

Fig. 8. Average percentage of substitutions processed by PScore_LP and PScore_NWST w.r.t. PScore_Base.

compensate for its longer computation time. In large tree and large graph queries, PScore_LP takes much longer to compute the partial PM-queries, and their better quality appears to have less impact than the longer computation times. Wrapping up, PScore_NWSTappears to be the best choice for tree-structured and larger queries, whereas PScore_LPis better suited for small graph queries. Interestingly, in the majority of cases the performance advantage of PScore_NWST over PScore_LP slightly increases (around 2\%) when $\gamma=0.7$.

Moreover, we specifically assessed the benefits provided by the use of the PM_Index as well as the quality of the upper bounds computed using the index, as described in Section 5.2. Figure 9 shows the performance advantage obtained when we precompute the PM_Index (which is the default case in this experimental assessment) w.r.t. the case where the information provided by the index is computed on-the-fly. The results confirm noticeable benefits: the advantage is around $17.5 \%$ on average, and more than $30 \%$ in some cases on the CiteSeerX, IMDb, YouTube, and Flickr datasets.

Figure 10 shows that the ratios between upper bounds and real scores of the substitutions processed are satisfactory (under 1.5 in the majority of cases).

In addition, to verify that the PScore algorithm quickly finds out that there is no top- $k$ answer to be returned when the number of substitutions is lower than $k$ (as discussed in Section 5.4), we ran a modified version of the queries on the Orkut dataset. In these queries, we replaced a constant with one that is not present in the dataset-the other parameters were those used for the original experiments (Figure 7). Evaluation times were indeed extremely low, never exceeding 2 seconds. As expected, PScore_NWST was faster than PScore_LP as it saved additional time when computing partial PM-queries.

Our second round of experiments (Figure 11) assessed how the performance of PScore_NWST and PScore_LP varies with the value of $k$. We measured query evaluation times obtained for different values of $k$ and $\gamma$, using annotated single-label queries. As expected, since pruning happens later when $k$ increases, evaluation times increase with $k$. Both our algorithms scale gracefully with $k$, and PScore_NWST is faster than PScore_LP in the majority of cases with $\gamma=0.3$ and in all cases 
Nuclear

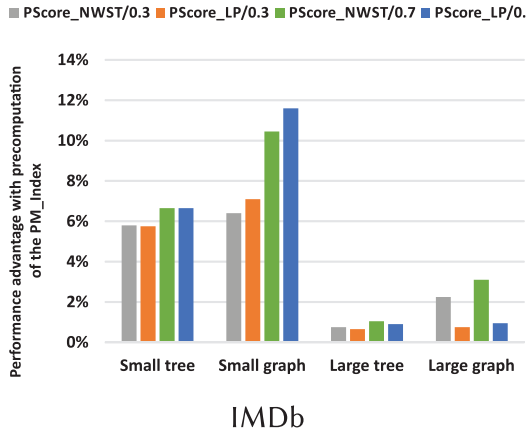

|| PScore_LP/0.3 $\mid$ PScore_NWST/0.3 $=$ PScore_LP/0.7 $=$ PScore_NWST/0.7

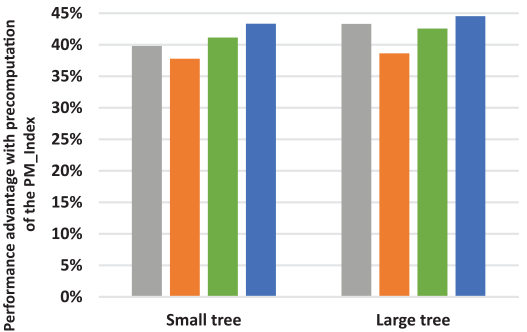

Flickr

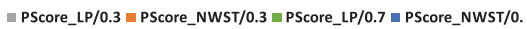

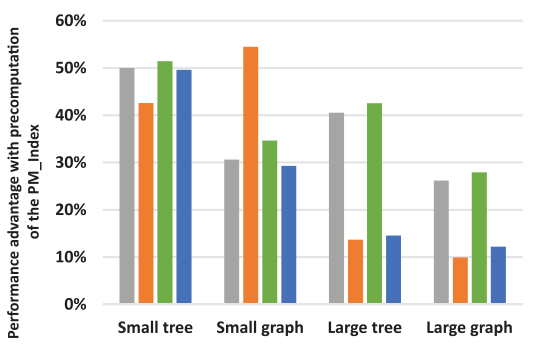

CiteSeerX

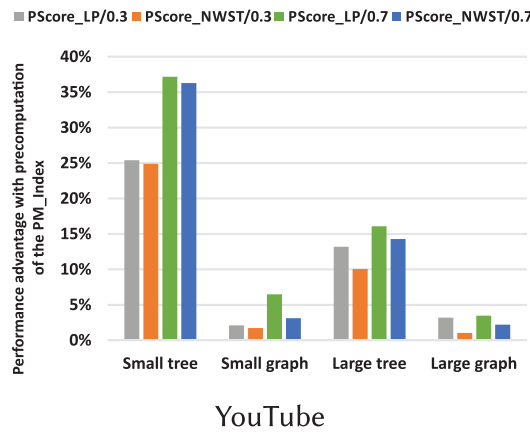

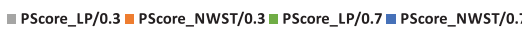

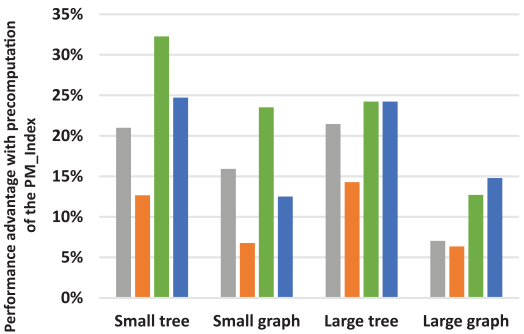

Orkut

| PScore_NWST/0.3 | PScore_LP/0.3 | PScore_NWST/0.7 | PScore_LP/0.7

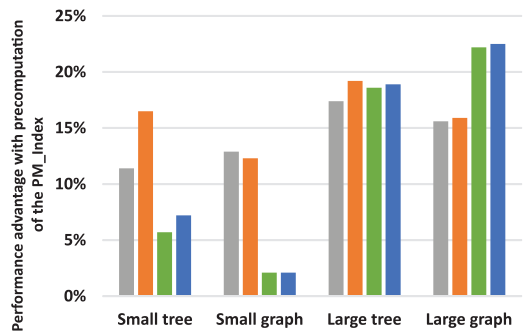

\section{BSBM}

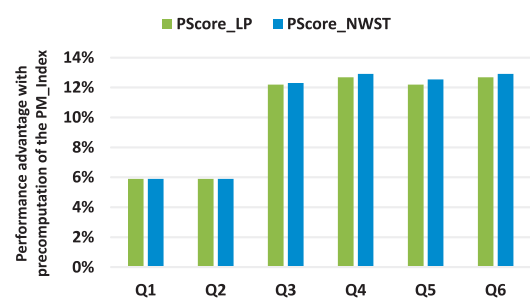

Fig. 9. Performance advantage with precomputation of the PM_Index. 


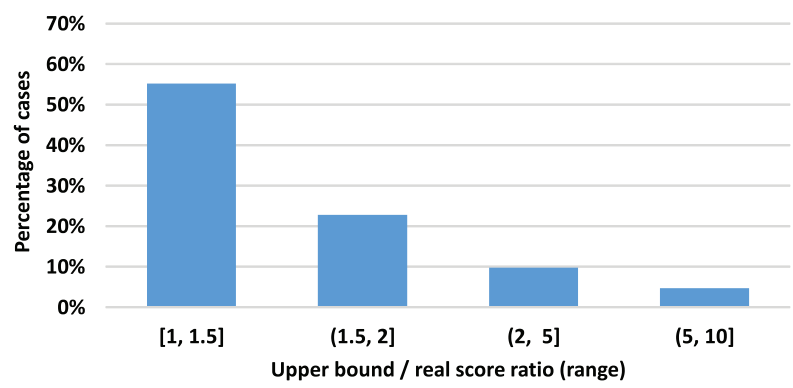

Fig. 10. Average ratio between upper bounds and real scores of the substitutions processed by PScore_LP and PScore_NWST.

with $\gamma=0.7$. PScore_Base, GraphDB and Jena do not prune, so their times cannot vary significantly w.r.t. $k$-on average, the performance advantage of PScore_NWST over GraphDB was $85.1 \%$.

In a third round of experiments, we assessed how the performance of PScore_NWST varies w.r.t. different query groups (non-annotated/single-label, annotated/single-label, annotated/multiplelabel). The results we obtained with $k=10$ on the Flickr dataset (that is the largest dataset with multiple labels for which we built synthetic queries) are reported in Figure 12. As expected, the impact of adding annotations and multiple labels is higher when $\gamma=0.3$, due to the higher number of substitutions the algorithm must handle.

Finally, we also measured the size and the build time of the PM_Index for different values of maximum distance. The results are reported in Table $8 .{ }^{13}$ Here, we can observe that the size of the index (whose increase w.r.t. distance depends on the average distance between vertices) "stabilizes" at a value of maximum distance around 3. Build times appear satisfactory if we consider the size of the datasets ${ }^{14}$ and, more importantly, the fact that the PM_Index (if used) is likely to be built in an offline fashion.

\subsection{Quality of the Answers}

We performed additional experiments to assess the quality of the answers provided by our framework, as perceived by human users.

We provided 10 users with an anonymized version of the Nuclear dataset and asked them to express their preferences about the answers computed using (1) our query model, (2) a model that finds top- $k$ substitutions based on a user-defined scoring function and does not perform aggregations (such as those in References $[32,39]$ ), ${ }^{15}$ and (3) a model that performs inexact matching based on wildcards or mutivalued attributes (such as that in Reference [41]).

To achieve this, we defined the following five questions and translated them into queries in each query model (a graphical representation of the S-queries obtained this way is reported in the Appendix). For each question, the users were asked to evaluate the quality of the results by stating their preferences with respect to the three top-five answer sets computed using the above query models.

$Q_{1}$ Which member of a given very large terrorist group has the most significant connections to other suspicious individuals?

\footnotetext{
${ }^{13} h \geq 4$ was not needed for the BSBM queries.

${ }^{14}$ In particular, the construction of the PM_Index has shown an approximate average case complexity of $O(|E|)$ in our experiments.

${ }^{15}$ This model actually corresponds to the SPARQL query model with ORDER BY.
} 
Nuclear

| PScore_LP/0.3 $\square$ PScore_NWST/0.3 $\square$ PScore_LP/0.7 $\square$ PScore_NWST/0.7

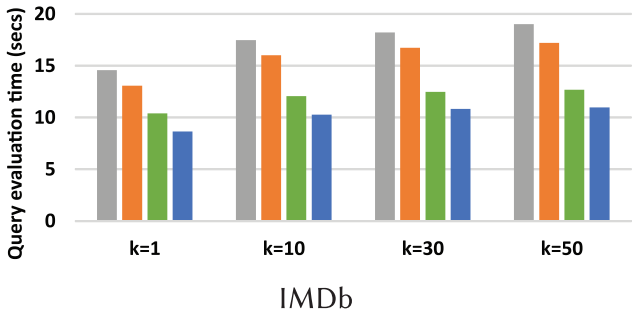

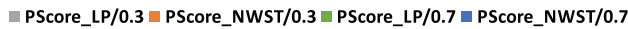

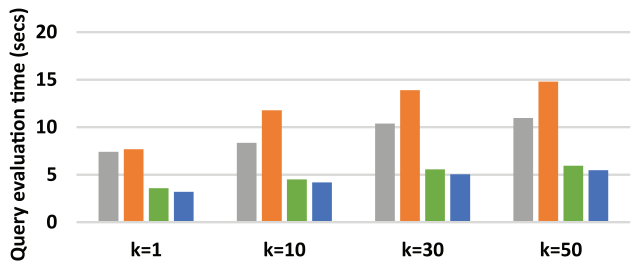

Flickr

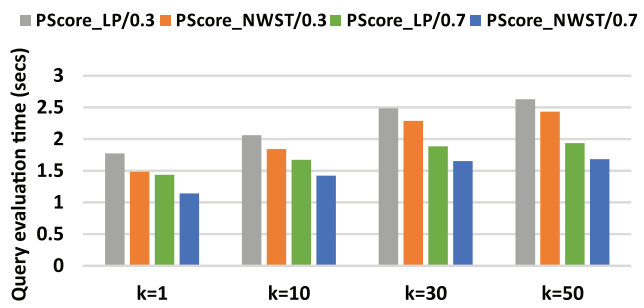

CiteSeerX

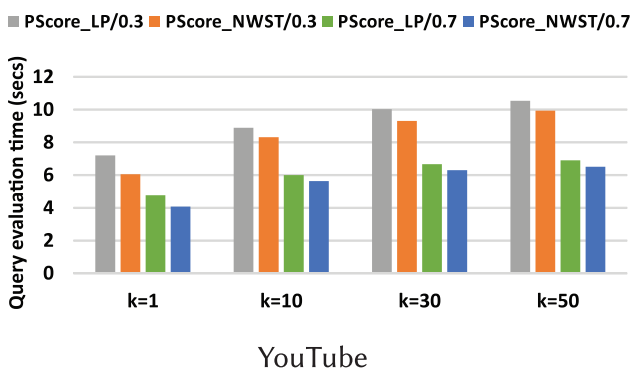

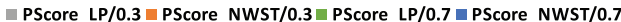

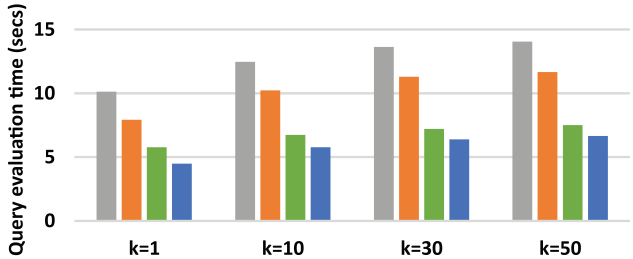

Orkut

$\llbracket$ PScore_LP/0.3 $₫$ PScore_NWST/0.3 $₫$ PScore_LP/0.7 $\backsim$ PScore_NWST/0.7

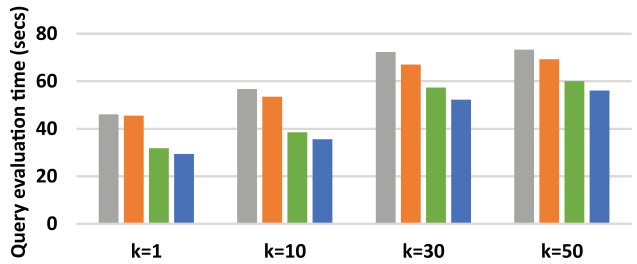

BSBM

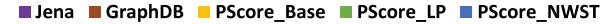

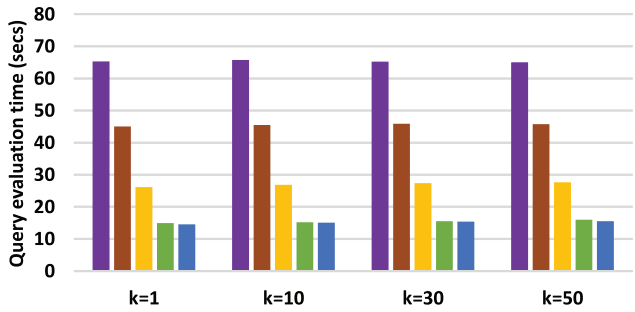

Fig. 11. Query evaluation time for different algorithms and values of $k$ and $\gamma$.

$Q_{2}$ Which of a given holding company's partner companies has the most significant connections with other suspicious individuals or companies?

$Q_{3}$ Which company has the most significant connections with a given terrorist who is a primary suspect in a well-known attack, and to his friends?

$Q_{4}$ Who is the key person connecting a given government intelligence agency, which has a large number of connections to many individuals, with other government agencies? 


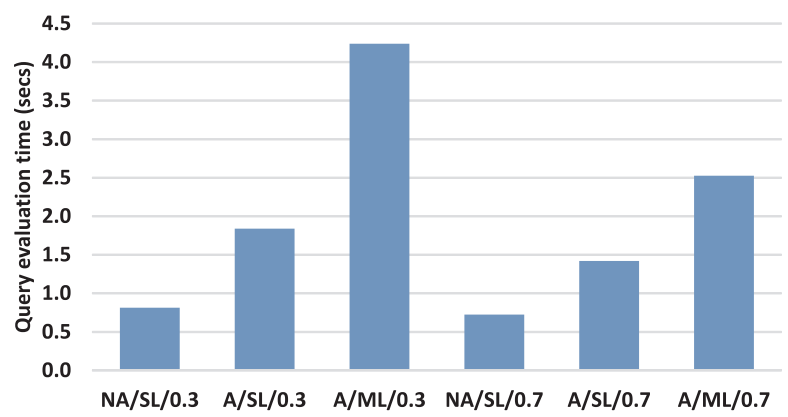

Fig. 12. Query evaluation time for different query groups and values of $\gamma$. Label $X / Y / x$ means query group $X / Y$ with $\gamma=x$. Annotated (resp. non-annotated) queries are denoted as A (resp. NA). Single-label (resp. multiple-label) queries are denoted as SL (resp. ML).

Table 8. Size (Number of Rows) and Build Time of the PM_Index for Different Values of Maximum Distance

\begin{tabular}{|c|c|c|c|c|c|c|}
\hline & 1 & 2 & 3 & 4 & 5 & Time \\
\hline \hline Nuclear & $0.52 \mathrm{M}$ & $0.9 \mathrm{M}$ & $1.29 \mathrm{M}$ & $1.5 \mathrm{M}$ & $2.38 \mathrm{M}$ & $5 \mathrm{mins}$ \\
CiteSeerX & $2.15 \mathrm{M}$ & $3.94 \mathrm{M}$ & $5.17 \mathrm{M}$ & $5.84 \mathrm{M}$ & $6.08 \mathrm{M}$ & $8 \mathrm{mins}$ \\
IMDb & $2.51 \mathrm{M}$ & $8.81 \mathrm{M}$ & $14.02 \mathrm{M}$ & $22.31 \mathrm{M}$ & $24.46 \mathrm{M}$ & $40 \mathrm{mins}$ \\
YouTube & $5.94 \mathrm{M}$ & $9.95 \mathrm{M}$ & $11.25 \mathrm{M}$ & $11.31 \mathrm{M}$ & $11.31 \mathrm{M}$ & $19 \mathrm{mins}$ \\
Flickr & $8.94 \mathrm{M}$ & $23.60 \mathrm{M}$ & $35.06 \mathrm{M}$ & $35.74 \mathrm{M}$ & $36.04 \mathrm{M}$ & $59 \mathrm{mins}$ \\
BSBM & $30.33 \mathrm{M}$ & $109.72 \mathrm{M}$ & $151.62 \mathrm{M}$ & - & - & $28 \mathrm{mins}$ \\
Orkut & $24.1 \mathrm{M}$ & $96.89 \mathrm{M}$ & $110.01 \mathrm{M}$ & $112.34 \mathrm{M}$ & $114.2 \mathrm{M}$ & $78 \mathrm{mins}$ \\
\hline
\end{tabular}

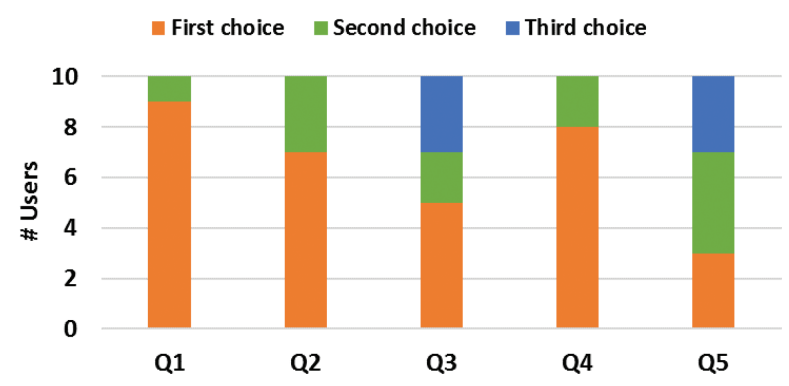

Fig. 13. User-perceived quality of the answers provided by our framework.

$Q_{5}$ Which company among all those connected to a given banking company in Asia has the most significant connections to suspicious individuals?

Figure 13 summarizes how the results computed using our proposed query model were ranked. In particular, for each query, the figure reports the number of users that stated that the answer set provided by our framework was their first, second, or third choice.

The results appear very satisfactory. In 32 out of 50 cases, our query model was identified as the one that provides the best answers (first choice)-in 12 cases, it was ranked as the second choice. In addition, (i) for all queries but $Q_{5}$, at least half of the users stated that our model was their first choice and (ii) for all queries, at least seven users stated that our model was their first or second choice. 
It should also be observed that, in general, the perceived quality of the answers also depends on the specific way natural-language questions are translated into graph queries. In our experiments, the users were only made aware of the questions and then looked directly at the results (not at the actual graph queries). For instance, if we consider $Q_{5}-$ "Which company among all those connected to a given banking company in Asia has the most significant connections to suspicious individuals?"-the corresponding graph-based query captures just one specific way of defining "significant connections." We believe that, with an in-depth knowledge of the application domain, and possibly after exchanging information with the users about the specific semantics of the questions they are interested in, the perceived quality of the answers can be further improved.

\section{CONCLUSIONS}

Edge-labeled graphs are occurring increasingly frequently. In many applications, it is appropriate to allow a user to specify functions that capture what is important for her because any systemdefined function would necessarily not take application-specific knowledge into account. For instance, how a nuclear proliferation expert assesses a vertex to be suspicious would differ from how an MIF investigator assesses a vertex's level of suspicion. In this article, we argue that the user may express her notion of importance via a set of subgraph patterns called PM-queries, and propose two query processing algorithms that leverage the notion of "partial PM-queries." We carried out detailed experiments with real-world graph databases with up to 234M edges. Our experiments show that answers on these real-world datasets can be computed in very good time frames.

\section{APPENDICES}

\section{A PROOF OF THEOREM 5.5}

Claim: Suppose $P Q=\left\langle V_{P Q}, E_{P Q}, \chi, \varrho, l a\right\rangle$ is a PM-query. Then

$$
\operatorname{sub}^{*}(P Q)=\prod_{? x \in V_{P Q} \cap V A R} \operatorname{cand}^{*}(P Q, ? x)
$$

is an upper bound on the number of substitutions for $P Q$.

Proof. We start by observing that any PM-query $P Q$ can be decomposed into $h=\left|V \cap V_{P Q}\right|$ disjoint PM-queries $S T\left(c_{1}\right), S T\left(c_{2}\right), \ldots, S T\left(c_{h}\right)$ such that each $S T\left(c_{i}\right)$ is a sub-tree of $P Q$ rooted at constant vertex $c_{i}$ (for simplicity, we do not consider $\chi$ and $\varrho$ here). The set of answer substitutions for each $S T(c)$, denoted $\Theta(c)$, contains all the substitutions that can be obtained by projecting answer substitutions for $P Q$ over the variables in $S T(c)$. Thus, an upper bound on the number of substitutions for $P Q$ is $\prod_{c \in V \cap V_{P Q}}|\Theta(c)|$.

Now, for each constant $c$, let $V A R_{c}$ be the set of variables ?x that are closest to $c$ and, therefore, whose $\operatorname{cand}^{*}(P Q, ? x)$ values are assigned based on the shortest path between $c$ and $? x$ (by definition of $c a n d^{*}(P Q, ? x)$ ). The set of shortest paths from $c$ to each $? x \in V A R_{c}$ constitutes a tree rooted at $c$. If we use this tree as our $S T(c)$, an upper bound on $|\Theta(c)|$ can easily be computed by taking the product of the $\operatorname{cand}^{*}(P Q, ? x)$ values of all variables in $S T(c)$. Thus, we can derive $\prod_{c \in V \cap V_{P Q}}|\Theta(c)| \leq \prod_{c \in V \cap V_{P Q}} \prod_{? x \in V_{S T(c)} \cap V A R}$ cand $^{*}(S T(c), ? x)=$ $\prod_{? x \in V_{P Q} \cap V A R} \operatorname{cand}^{*}(P Q, ? x)$. The statement follows. 


\section{B PROOF OF THEOREM 5.7}

Claim: opt $(P Q)_{\log }^{B}$ is NP-complete.

Proof. Membership in NP is trivial. We show NP-hardness by reduction from the Steiner tree problem [17] defined as follows. Given an undirected graph $\widehat{G}=\langle\widehat{V}, \widehat{E}\rangle$ a subset of vertices $S \subseteq \widehat{V}$ called terminals, and a positive integer $K \leq|\widehat{V}|-1$, decide whether there is a subtree of $\widehat{G}$ that includes all the vertices in $S$ (i.e., a spanning tree for $S$ ) and contains no more than $K$ edges. Given an instance of ST, we construct an instance of our problem as follows. Let $B=|\widehat{V}|-(K+1)$, and $P Q=\left\langle V_{P Q}, E_{P Q}, \chi, \varrho, l a\right\rangle$ be such that (i) $V_{P Q}$ consists of a variable $? v$ for each vertex $v \in \widehat{V}$, plus an additional constant vertex $c$; (ii) $E_{P Q}$ consists of an edge $\left(? v_{1},\{\ell\}, 1, ? v_{2}\right)$ for each edge $\left(v_{1}, v_{2}\right) \in \widehat{E}$, plus an additional edge $\left(? v^{*},\{\ell\}, 1, c\right)$ where $v^{*}$ is any vertex in $S$ and $\ell$ is any edge label; (iii) $\chi$ is empty; (iv) $\forall v \in S \varrho(? v)=t$, where $t$ is any numerical term; (v) la is any aggregation function. Moreover, assume that $\forall ? v \in V_{P Q}, \operatorname{cand}^{*}(P Q, ? v)=2$ (it can be easily shown that there always exist a graph database such that $\operatorname{cand}(c, \delta, \pi, e)=2$, where $c$ is the constant vertex of $P Q$ ).

Observe that each variable ? $v$ corresponding to a vertex $v \in S$ is associated with a numerical term by $\varrho$, so ? $v$ is an output variable of $P Q$, and thus it belongs to the set of essential variables, i.e., $\mathcal{E}(P Q)=\{? v \mid v \in S\}$.

We now show that that there is a tree $T=\left\langle\widehat{V}_{T}, \widehat{E}_{T}\right\rangle$ of $\widehat{G}$ that includes all terminals and contains no more than $K$ edges iff there is a partial $P M$-query $P P Q$ for $P Q$ such that $\log _{2} s u b^{*}(P P Q, P Q) \geq B$.

$(\Rightarrow)$ Given such a tree $T=\left\langle\widehat{V}_{T}, \widehat{E}_{T}\right\rangle$, we define $P P Q(T)$ such that $V_{P P Q(T)}=\widehat{V}_{T} \cup\{c\}$ and $E_{P P Q(T)}=\widehat{E}_{T} \cup\left\{\left(? v^{*},\{\ell\}, 1, c\right)\right\}$. Clearly $P P Q(T)$ is a partial PM-query, as there exists at least one path between every pair of essential variables in $\mathcal{E}(P Q)$ (since there is one path in $T$ between each pair of terminals), and at least one path between each essential variable in $\mathcal{E}(P Q)$ and the constant vertex $c \in V_{P P Q(T)}$ (in fact, $c$ is connected to essential variable ? $v^{*}$ by $\left(? v^{*},\{\ell\}, 1, c\right) \in E_{P P Q(T)}$, and $? v^{*}$ is connected to the other essential variables of $\left.P Q\right)$. Moreover, $P P Q(T)$ is such that $\left|? v \in\left(V_{P Q} \backslash V_{P P Q(T)}\right) \cap V A R\right|=|\widehat{V}|-(K+1)$. Thus, $\log _{2}\left(\prod_{? x \in\left(V_{P Q} \backslash V_{P P Q(T)}\right) \cap V A R} \operatorname{cand}^{*}(P Q, ? x)\right)=$ $\sum_{? x \in\left(V_{P Q} \backslash V_{P P Q(T)}\right) \cap V A R} \log _{2} \operatorname{cand}^{*}(P Q, ? x)=\left|? x \in\left(V_{P Q} \backslash V_{P P Q(T)}\right) \cap V A R\right| \geq B$.

$(\Leftarrow)$ Assume now that there is a partial $P M$-query $P P Q$ for $P Q$ such that $\log _{2}$ sub $(P P Q, P Q) \geq B$. Thus, there is subgraph $\left\langle V_{P P Q}, E_{P P Q}\right\rangle$ of $\left\langle V_{P Q}, E_{P Q}\right\rangle$ such that (i) $\{c\} \cup \mathcal{E}(P Q) \subseteq V_{P P Q}$, (ii) there is least one path between each essential variable in $\mathcal{E}(P Q)$, (iii) there is at least one path between each essential variable in $\mathcal{E}(P Q)$ and the constant vertex $c \in V_{P P Q}$, and (iv) $\log _{2}$ sub $b^{*}(P P Q, P Q)=\mid ? x \in$ $\left(V_{P Q} \backslash V_{P P Q(T)}\right) \cap V A R \mid \geq B$. Let $G(P P Q)=\langle V(P P Q), E(P P Q)\rangle$ be the subgraph of $\widehat{G}$ such that $V(P P Q)=\left\{v \mid ? v \in V_{P P Q} \cap V A R\right\}$ and $E(P P Q)=\left\{\left(v_{1}, v_{2}\right) \mid\left(? v_{1},\{\ell\}, 1, ? v_{2}\right) \in E_{P P Q}\right.$ and $? v_{1}, ? v_{2} \in$ $V(P P Q)\}$. Clearly, $|V(P P Q)|=\left|V_{P P Q} \cap V A R\right| \leq\left|V_{P Q} \cap V A R\right|-B=|\widehat{V}|-(|\widehat{V}|-(K+1))=K+1$. Hence, if $G(P P Q)$ is a tree, we are done, since it is a subtree of $\widehat{G}$ that includes all vertices in $S$ and contains no more than $K$ edges. Otherwise, a spanning tree $T(P P Q)$ for the whole set of vertex $V(P P Q)$ of $G(P P Q)$ can be constructed in polynomial time by removing some edges from $E(P P Q)$. This way a subtree $T(P P Q)$ of $\widehat{G}$ that includes all terminals and contains no more than $K+1$ vertices (and thus $K$ edges) is obtained as well.

\section{PROOF OF THEOREM 5.9}

Claim: Given a PM-query $P Q$, every solution $T$ of $N W S T(P Q)$ one-to-one corresponds to a partial PM-query $P P Q(T)$ for $P Q$ such that $s u b^{*}(P P Q(T), P Q)$ is maximum. 
Proof. Let $T$ be a minimum weight subtree $T=\left\langle\widehat{V}_{T}, \widehat{E}_{T}\right\rangle$ of $N W S T(P Q)$ such that $T$ includes all the vertices in $S$. Let $P P Q(T)$ be such that $V_{P P Q(T)}=\widehat{V}_{T}$ and $E_{P P Q(T)}=\widehat{E}_{T}$.

Clearly, Definition 5.1 holds for $P P Q(T)$, as there is one path in $T$ between each pair of vertices in $S=\mathcal{E}(P Q) \cup\left(V_{P Q} \cap V\right)$. Observe that $P P Q(T)$ includes all the constant vertices of $P Q$. However, the value $s u b^{*}(P P Q(T), P Q)$ does not depend on this.

We now show that $s u b^{*}(P P Q(T), P Q)$ is maximum. Let $V^{*}$ be the set $V_{P P Q(T)} \cap V A R$. The fact that $\sum_{v \in V_{T}} \omega(v)$ is minimum entails that $\sum_{? x \in V^{*}} \omega(? x)=\sum_{? x \in V^{*}} \ln \operatorname{cand}^{*}(P Q, ? x)=\ln \prod_{? x \in V^{*}}$ cand $^{*}$ $(P Q, ? x)$ is minimum too. Therefore, $\ln s u b^{*}(P P Q(T), P Q)=$

$$
\begin{gathered}
\ln \prod_{? x \in\left(V_{P Q} \backslash V_{P P Q}(T) \cap V A R\right.} \operatorname{cand}^{*}(P Q, ? x)= \\
\ln \frac{\prod_{? x \in V_{P Q^{\cap} V A R}} \operatorname{cand}^{*}(P Q, ? x)}{\prod_{? x \in V^{*}} \operatorname{cand}^{*}(P Q, ? x)} \\
\ln \prod_{? x \in V_{P Q} \cap V A R} \operatorname{cand}^{*}(P Q, ? x)-\ln \prod_{? x \in V^{*}} \operatorname{cand}^{*}(P Q, ? x)
\end{gathered}
$$

is maximized, as the subtrahend is minimized. 


\section{QUERIES USED IN SECTION 7.3}

The queries used in the evaluation of the quality of the answers are reported below.

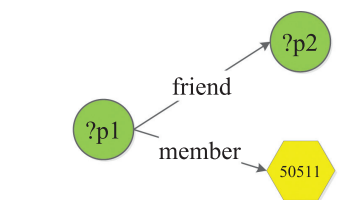

$\operatorname{scr}(? \mathrm{p} 1)=? \mathrm{p} 1 . \mathrm{sus}+(1+\text { ?p } 2 . \text { sus })^{\wedge} 2$

(a) $Q_{1}$

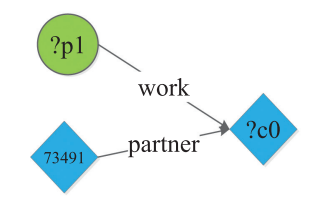

$\operatorname{scr}(? \mathrm{c} 0)=? \mathrm{c} 0 . \mathrm{sus}+(1+? \mathrm{p} 1 . \mathrm{sus})^{\wedge} 2$

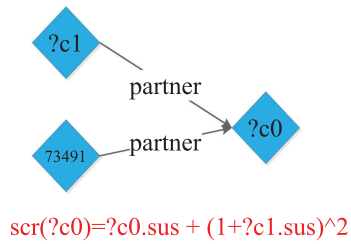

(b) $Q_{2}$

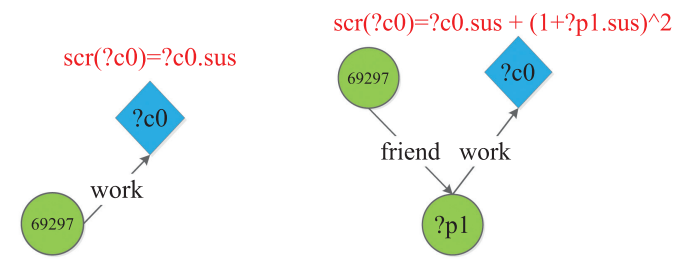

(c) $Q_{3}$

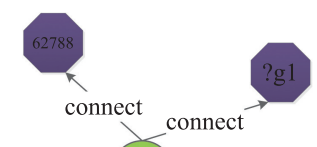

?p1

$\operatorname{scr}(? \mathrm{p} 1)=$ ?p1.sus $+(1+\text { ?g } 1 . \text { sus })^{\wedge} 2$

(d) $Q_{4}$

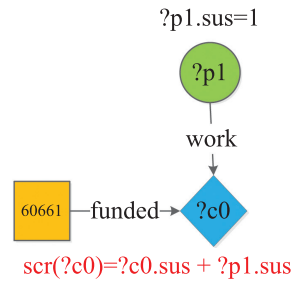

(e) $Q_{5}$ 


\section{REFERENCES}

[1] Ian A. Andrews, Srijan Kumar, Francesca Spezzano, and V. S. Subrahmanian. 2015. SPINN: Suspicion prediction in nuclear networks. In IEEE International Conference on Intelligence and Security Informatics (ISI). 19-24.

[2] Marcelo Arenas, Sebastián Conca, and Jorge Pérez. 2012. Counting beyond a yottabyte, or how SPARQL 1.1 property paths will prevent adoption of the standard. In International World Wide Web Conference (WWW). 629-638.

[3] Medha Atre, Vineet Chaoji, Mohammed J. Zaki, and James A. Hendler. 2010. Matrix "Bit" loaded: A scalable lightweight join query processor for RDF data. In International World Wide Web Conference (WWW). 41-50.

[4] Medha Atre, Jagannathan Srinivasan, and James A. Hendler. 2008. BitMat: A main-memory bit matrix of RDF triples for conjunctive triple pattern queries. In International Semantic Web Conference Posters and Demos.

[5] Barry Bishop, Atanas Kiryakov, Damyan Ognyanoff, Ivan Peikov, Zdravko Tashev, and Ruslan Velkov. 2011. OWLIM: A family of scalable semantic repositories. Semantic Web 2, 1 (2011), 33-42.

[6] Christian Bizer and Andreas Schultz. 2009. The Berlin SPARQL benchmark. Int. f. Semantic Web Inf. Syst. 5, 2 (2009), $1-24$.

[7] Matthias Bröcheler, Andrea Pugliese, and V. S. Subrahmanian. 2009. DOGMA: A disk-oriented graph matching algorithm for RDF databases. In International Semantic Web Conference. 97-113.

[8] Jeremy J. Carroll, Ian Dickinson, Chris Dollin, Dave Reynolds, Andy Seaborne, and Kevin Wilkinson. 2004. Jena: Implementing the semantic web recommendations. In International World Wide Web Conference (WWW). 74-83.

[9] Meeyoung Cha, Alan Mislove, and P. Krishna Gummadi. 2009. A measurement-driven analysis of information propagation in the Flickr social network. In International World Wide Web Conference (WWW). 721-730.

[10] Jiefeng Cheng and Jeffrey Xu Yu. 2011. A survey of relational approaches for graph pattern matching over large graphs. In Graph Data Management. 112-141.

[11] Jiefeng Cheng, Xianggang Zeng, and Jeffrey Xu Yu. 2013. Top-k graph pattern matching over large graphs. In IEEE International Conference on Data Engineering. 1033-1044.

[12] Xu Cheng, Cameron Dale, and Jiangchuan Liu. 2008. Statistics and social network of youtube videos. In IEEE/ACM International Symposium on Quality of Service (IWQoS). 229-238.

[13] CiteSeerX. 2016. Public dataset. Retrieved March 15, 2018 from http://csxstatic.ist.psu.edu/about/data.

[14] Shady Elbassuoni, Maya Ramanath, and Gerhard Weikum. 2012. RDF Xpress: A flexible expressive RDF search engine. In International ACM SIGIR Conference on Research and Development in Information Retrieval. 1013.

[15] Wenfei Fan, Xin Wang, and Yinghui Wu. 2013. Diversified top-k graph pattern matching. PVLDB 6, 13 (2013), 15101521.

[16] Yuan Fang, Wenqing Lin, Vincent Wenchen Zheng, Min Wu, Kevin Chen-Chuan Chang, and Xiaoli Li. 2016. Semantic proximity search on graphs with metagraph-based learning. In IEEE International Conference on Data Engineering. $277-288$

[17] Michael R. Garey and David S. Johnson. 1979. Computers and Intractability: A Guide to the Theory of NP-Completeness. W. H. Freeman \& Co., New York.

[18] Gang Gou and Rada Chirkova. 2008. Efficient algorithms for exact ranked twig-pattern matching over graphs. In ACM SIGMOD Conference. 581-594.

[19] GraphDB. 2016. Home page. Retrieved March 15, 2018 from http://www.ontotext.com.

[20] Alvaro Graves, Sibel Adali, and Jim Hendler. 2008. A method to rank nodes in an RDF graph. In International Semantic Web Conference Posters and Demos.

[21] Sudipto Guha and Samir Khuller. 1999. Improved methods for approximating node weighted Steiner trees and connected dominating sets. Inf. Comput. 150, 1 (1999), 57-74.

[22] Wook-Shin Han, Jinsoo Lee, Minh-Duc Pham, and Jeffrey Xu Yu. 2010. iGraph: A framework for comparisons of disk-based graph indexing techniques. PVLDB (2010).

[23] Steve Harris and Andy Seaborne. 2016. SPARQL 1.1 Query Language. Retrieved March 15, 2018 from http://www.w3. org/TR/sparql11-query.

[24] Zhipeng Huang, Yudian Zheng, Reynold Cheng, Yizhou Sun, Nikos Mamoulis, and Xiang Li. 2016. Meta structure: Computing relevance in large heterogeneous information networks. In SIGKDD Conference on Knowledge Discovery and Data Mining. 1595-1604.

[25] IMDb. 2016. Public dataset. Retrieved March 15, 2018 from http://www.imdb.com/interfaces.

[26] Jena. 2016. Home page. Retrieved March 15, 2018 from https://jena.apache.org.

[27] Foteini Katsarou, Nikos Ntarmos, and Peter Triantafillou. 2015. Performance and scalability of indexed subgraph query processing methods. PVLDB (2015).

[28] Arijit Khan, Yinghui Wu, Charu C. Aggarwal, and Xifeng Yan. 2013. NeMa: Fast graph search with label similarity. PVLDB 6, 3 (2013), 181-192.

[29] Jinsoo Lee, Wook-Shin Han, Romans Kasperovics, and Jeong-Hoon Lee. 2013. An in-depth comparison of subgraph isomorphism algorithms in graph databases. In Proceedings of the International Conference on Very Large Data Bases $(V L D B)$

ACM Transactions on the Web, Vol. 12, No. 4, Article 21. Publication date: September 2018. 
[30] Lehigh University Benchmark (LUBM). 2016. Home page. Retrieved March 15, 2018 from http://swat.cse.lehigh.edu/ projects/lubm.

[31] Wenqing Lin, Xiaokui Xiao, James Cheng, and Sourav S. Bhowmick. 2012. Efficient algorithms for generalized subgraph query processing. In ACM International Conference on Information and Knowledge Management. 325-334.

[32] Sara Magliacane, Alessandro Bozzon, and Emanuele Della Valle. 2012. Efficient execution of top-k SPARQL queries. In International Semantic Web Conference. 344-360.

[33] Alan Mislove, Massimiliano Marcon, P. Krishna Gummadi, Peter Druschel, and Bobby Bhattacharjee. 2007. Measurement and analysis of online social networks. In ACM SIGCOMM Conference. 29-42.

[34] Mohamed Morsey, Jens Lehmann, Sören Auer, and Axel-Cyrille Ngonga Ngomo. 2011. DBpedia SPARQL benchmark-Performance assessment with real queries on real data. In International Semantic Web Conference. 454469.

[35] Lorenzo De Nardo, Francesco Ranzato, and Francesco Tapparo. 2009. The subgraph similarity problem. IEEE Trans. Knowl. Data Eng. 21, 5 (2009), 748-749.

[36] Neo4j. 2016. Home page. Retrieved March 15, 2018 from http://neo4j.com.

[37] Thomas Neumann and Gerhard Weikum. 2010. The RDF-3X engine for scalable management of RDF data. VLDB $\mathcal{F}$. 19, 1 (2010), 91-113.

[38] Thomas Neumann and Gerhard Weikum. 2010. x-RDF-3X: Fast querying, high update rates, and consistency for RDF databases. PVLDB 3, 1 (2010), 256-263.

[39] Michael Ovelgönne, Noseong Park, V. S. Subrahmanian, Elizabeth K. Bowman, and Kirk Ogaard. 2013. Personalized best answer computation in graph databases. In International Semantic Web Conference.

[40] Zhengxiang Pan, Xingjian Zhang, and Jeff Heflin. 2008. DLDB2: A scalable multi-perspective semantic web repository. In International Conference on Web intelligence (WI). 489-495.

[41] Robert Pienta, Acar Tamersoy, Hanghang Tong, and Duen Horng Chau. 2014. MAGE: Matching approximate patterns in richly-attributed graphs. In IEEE International Conference on Big Data. 585-590.

[42] Yan Qi, K. Selçuk Candan, and Maria Luisa Sapino. 2007. Sum-max monotonic ranked joins for evaluating top-k twig queries on weighted data graphs. In International Conference on Very Large Data Bases (VLDB). 507-518.

[43] Sherif Sakr and Ghazi Al-Naymat. 2009. Relational processing of RDF queries: A survey. ACM SIGMOD Conference Rec. 38, 4 (2009), 23-28.

[44] Chuan Shi, Yitong Li, Jiawei Zhang, Yizhou Sun, and Philip S. Yu. 2017. A survey of heterogeneous information network analysis. IEEE Trans. Knowl. Data Eng. 29, 1 (2017), 17-37.

[45] Social Network Intelligence Benchmark. 2016. Home page. Retrieved March 15, 2018 from http://www.w3.org/wiki/ Social_Network_Intelligence_BenchMark.

[46] SP ${ }^{2}$ Bench. 2016. Home page. Retrieved March 15, 2018 from http://dbis.informatik.uni-freiburg.de/forschung/ projekte/SP2B.

[47] SPARQLer. 2016. Home page. Retrieved March 15, 2018 from http://www.sparql.org.

[48] Yizhou Sun, Jiawei Han, Xifeng Yan, Philip S. Yu, and Tianyi Wu. 2011. PathSim: Meta path-based top-k similarity search in heterogeneous information networks. PVLDB 4, 11 (2011), 992-1003.

[49] Zhao Sun, Hongzhi Wang, Haixun Wang, Bin Shao, and Jianzhong Li. 2012. Efficient subgraph matching on billion node graphs. PVLDB 5, 9 (2012), 788-799.

[50] Titan. 2016. Home page. Retrieved March 15, 2018 from http://thinkaurelius.github.io/titan.

[51] Kevin Wilkinson, Craig Sayers, Harumi A. Kuno, and Dave Reynolds. 2003. Efficient RDF storage and retrieval in jena2. In International Conference on Semantic Web and Databases. 131-150.

[52] Xifeng Yan, Bin He, Feida Zhu, and Jiawei Han. 2010. Top-k aggregation queries over large networks. In IEEE International Conference on Data Engineering. 377-380.

[53] Shengqi Yang, Fangqiu Han, Yinghui Wu, and Xifeng Yan. 2016. Fast top-k search in knowledge graphs. In IEEE International Conference on Data Engineering.

[54] Yuanyuan Zhu, Lu Qin, Jeffrey Xu Yu, and Hong Cheng. 2012. Finding top-k similar graphs in graph databases. In International Conference on Extending Database Technology. 456-467.

[55] Lei Zou, Lei Chen, and Yansheng Lu. 2007. Top-k subgraph matching query in a large graph. In Ph.D. Workshop on Information and Knowledge Management. 139-146.

[56] Lei Zou, M. Tamer Özsu, Lei Chen, Xuchuan Shen, Ruizhe Huang, and Dongyan Zhao. 2014. gStore: A graph-based SPARQL query engine. VLDB 7. 23, 4 (2014), 565-590.

Received May 2016; revised October 2017; accepted March 2018 